\title{
Elliptic Operators in Even Subspaces
}

\author{
Anton Savin* and Boris Sternin* \\ Moscow State University \\ e-mail: antonsavin@mtu-net.ru \\ e-mail: sternin@math.uni-potsdam.de
}

\begin{abstract}
An elliptic theory is constructed for operators acting in subspaces defined via even pseudodifferential projections. Index formulas are obtained for operators on compact manifolds without boundary and for general boundary value problems. A connection with Gilkey's theory of $\eta$-invariants is established.
\end{abstract}

Keywords: index of elliptic operators in subspaces, $K$-theory, eta invariant, AtiyahPatodi-Singer theory, boundary value problems

1991 AMS classification: Primary 58G03, Secondary 58G10, 58G12, 58G25, 19K56

*Supported by the Russian Foundation for Basic Research under grants No. 97-01-00703 and 97-0216722a, Arbeitsgruppe Partielle Differentialgleichungen und Komplexe Analysis, Institut für Mathematik, Universität Potsdam, and the International Soros Foundation. 


\section{Contents}

Introduction $\quad 2$

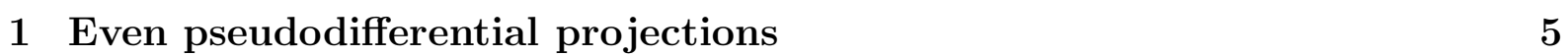

2 Operators in subspaces. An index formula for even subspaces 12

\begin{tabular}{lll}
\hline 3 & General boundary value problems & 18
\end{tabular}

\begin{tabular}{ll|l|l|l|}
4 & From general to spectral boundary value problems & 22
\end{tabular}

5 An index formula for even boundary value problems 27

\begin{tabular}{|lll}
6 & Eta invariants and even projections & 34
\end{tabular}

\begin{tabular}{lll}
\hline 7 & Examples & 37
\end{tabular}

\section{Introduction}

We study elliptic operators in subspaces defined by pseudodifferential projections, more precisely, even pseudodifferential projections. Such projections appear already in classical boundary value problems. Indeed, when we reduce an elliptic boundary value problem to the boundary, the corresponding operator acts in subspaces defined by pseudodifferential projections. Moreover, projections prove to be very helpful in the construction of a Fredholm theory for elliptic operators violating the well-known Atiyah-Bott condition [1]. It is shown in [2] that such a theory can be constructed in subspaces defined as the ranges of pseudodifferential projections in Sobolev spaces. These results actually go back to the classical Hardy spaces in which (and only in which) valid Fredholm theory for the Cauchy-Riemann operator can be constructed. The class of even projections is an important class of pseudodifferential projections. The precise definition will be given later, but for now we point out that in the subspaces defined by such projections we not only prove the finiteness theorem, but also present the corresponding index formula.

The most essential and fundamental distinction of elliptic theory in subspaces defined by pseudodifferential projections from a similar theory in Sobolev spaces is as follows. Although the ellipticity condition, just as in the classical case, is expressed in terms of the principal symbols of the main operator and the projections, the index of an elliptic operator in subspaces is determined by neither the principal nor even the complete symbols. This results in the necessity to give some numerical characteristic of pseudodifferential projections or, which is the same, of the subspaces they define. One can obtain such a numerical characteristic, playing in a certain sense the role of the dimension of a projection (in the finite-dimensional case it is equal to the rank of the projection), at 
least in the class of even projections. This notion is undoubtedly fundamental in our theory. In these terms we obtain an index formula in the situation of compact manifolds without boundary as well as for general boundary value problems. The relation between the notion of "dimension" and the $\eta$-invariant of Atiyah-Patodi-Singer [3] is established.

The paper is organized as follows.

In the first section, we consider subspaces defined as the ranges of pseudodifferential projections on an odd-dimesional compact manifold $M$ without boundary. More precisely, we assume that the projection is even, in the sense that its principal symbol is an even function with respect to the cotangent variables.

It turns out that in the class of such subspaces there exists a uniquely defined (up to a normalization) analog of the notion of dimension of a finite-dimensional vector space (i.e., a homotopy invariant additive functional). Every normalization is a choice of dimensions for spaces of sections of vector bundles over $M$.

Even projections have the following property: the group of stable homotopy classes of even projections is rationally generated by elements that differ from the projections on spaces of sections of vector bundles on $M$ by finite rank operators. This statement is actually a consequence of the fact that the obstruction to the stable homotopy of projections (modulo finite rank operators) lies in the group $K\left(P^{*} M\right) / K(M)$ (here $P^{*} M$ is the projectivization of the cosphere bundle), which is a torsion group.

In the second section we study elliptic operators acting in subspaces defined by pseudodifferential projections:

$$
D: H_{1} \longrightarrow H_{2}, \quad H_{1,2}=\operatorname{Im} P_{1,2} \text {. }
$$

Namely, we show that the index of elliptic operators of this form is represented as a sum of two homotopy invariant terms, one of which is determined by the principal symbol of the operator $D$ (and is a homotopy invariant of the principal symbol of the problem), while the second term is determined only by the subspaces where the operator $D$ acts and is a homotopy invariant of the subspaces.

Let us note that there is no decomposition of this kind in the class of all elliptic operators. It can be shown that here, as well as in the theory of spectral boundary value problems (see [ [4, 5]), there is an obstruction to such a decomposition, which is closely related to the spectral flows of periodic families of operators (see Sec. 1). That is why, to obtain an index formula, one must necessarily take narrower classes of operators.

In Sections 3, 4, and 5 we give an application of the theory to boundary value problems. The general boundary value problems [2] are considered in Sec. 3. They have he form

$$
\left\{\begin{array}{l}
D u=f, \\
B u=g \in \operatorname{Im} P,
\end{array}\right.
$$

${ }^{1}$ Let us note that all our constructions are determined by the subspaces themselves and are independent of the choice of projections onto them. 
where $D$ is an elliptic differential operator on a smooth manifold $M$ with boundary $\partial M$, $B$ is a boundary operator, and $P$ is some pseudodifferential projection on $\partial M$. This class of boundary value problems, on the one hand, contains all classical boundary value problems with the Lopatinskii condition (e.g., see [6]). On the other hand, for any elliptic operator $D$ there exists a Fredholm boundary value problem in this class. In this section all the necessary definitions are given. Examples are presented.

Sections 1 and 5 deal with the index computation for general boundary value problems. In Sec. [t the problem is reduced to a certain spectral boundary value problem [7]. In a collar neighborhood of the boundary with the normal coordinate $t$, it has the form

$$
\left\{\begin{array}{l}
\left(\frac{\partial}{\partial t}+A(t)\right) u=f, \\
\left.P u\right|_{\partial M}=g \in \operatorname{Im} P,
\end{array}\right.
$$

where $A(0)$ is a pseudodifferential operator on the boundary, whose homogeneous principal symbol on the bundle of cotangent spheres to the boundary is equal to

$$
\sigma(A(0))=2 \sigma(P)-1 .
$$

The reduction is understood in the sense that the corresponding Fredholm operators have the same indices.

In Sec. . the class of even boundary value problems on an even-dimensional manifold $M$ is considered. These are boundary value problems of the form (11) with an even projection $P$. Under this condition, we obtain the following index formula for the spectral boundary value problem (2):

$$
\text { ind }(D, P)=\frac{1}{2} \operatorname{ind}_{t}(\sigma(\tilde{D}))-d(P),
$$

where $\sigma(\tilde{D})$ is the elliptic symbol on the double of $M$ obtained by (continuously) gluing the symbols $\sigma(D)(\xi)$ and $\sigma(D)(-\xi)$, and $d(P)$ is the above-mentioned dimension functional for the trivial normalization. Let us note that, by virtue of the reduction carried out in the first part of the paper, formula (3) solves the index problem for general boundary value problems (11) in the case of even projections $P$.

The third application of the introduced notion of $d$-dimension is related to the $\eta$ invariant of Atiyah-Patodi-Singer [3]. Namely, the invariant $d$ of an even pseudodifferential projection is closely connected with the theory of $\eta$-invariants of self-adjoint elliptic operators of even order on odd-dimensional manifolds. More precisely, the $\eta$-invariant of an admissible operator [8] (see also Sec. [) in this case is equal to the introduced "dimension" of its nonnegative spectral subspace for the trivial normalization. It follows that the fractional parts of the "dimension" and of the $\eta$-invariant define a homomorphism

$$
K\left(P^{*} M\right) / K(M) \longrightarrow \mathbf{Z}\left[\frac{1}{2}\right] \bmod \mathbf{Z} .
$$

By virtue of this identification, the index formula (3) can be interpreted as an analog of the Atiyah-Patodi-Singer formula [3]. As a corollary to the index formula in subspaces, we 
obtain (see Corollary 11) a topological expression for the fractional part of the $\eta$-invariant on the subgroup

$$
\operatorname{ker}\left\{\pi^{*}: K\left(P^{*} M\right) / K(M) \longrightarrow K\left(S^{*} M\right) / K(M)\right\}, \quad \pi: S^{*} M \rightarrow P^{*} M .
$$

While the index formula for even boundary value problems shows the cobordism invariance of the fractional part of the doubled $\eta$-invariant (see Corollaries 2 and 3), it turns out that in both cases the $\eta$-invariant has at most 2 in the denominator or is even an integer. Nevertheless, the problem of the nontriviality of this fractional part remains open.

In the last section of the paper we consider several examples.

The authors are grateful to Prof. A. S. Mishchenko for a number of valuable remarks he made when this work was reported at his seminar in Moscow State University in fall 1998. He also suggested to include the applications concerning the boundary value problems, which undoubtedly improved the paper. We would like to thank V. E. Nazaikinskii for constructive suggestions that helped us improve the original version of the paper. Finally, we are grateful to Prof. P. Gilkey, the discussion with whom on the subject of this paper was extremely useful for us.

\section{Even pseudodifferential projections}

Let us consider the set $\operatorname{Proj}(M)$ of all pseudodifferential projections of order zero acting in the spaces of smooth sections of vector bundles on a closed manifold $M$. In this set it is impossible to compare the ranks (the dimensions of the range) of projections as one can do in the case of finite-dimensional spaces. More precisely, on the space of pseudodifferential projections there does not exist a homotopy invariant (with respect to the operator $L^{2}$-norm) functional

$$
d: \operatorname{Proj}(M) \longrightarrow \mathbf{Z} \quad(\text { or even } \mathbf{R} \text { or } \mathbf{C})
$$

that satisfies the (weak) additivity property

$$
d\left(P \oplus P^{\prime}\right)=d(P)+\operatorname{dim} \operatorname{Im} P^{\prime},
$$

for an arbitrary projection $P$, where $P^{\prime}$ is a finite-dimensional projection. Indeed, assuming the opposite, consider an arbitrary periodic family $A_{t}$ of self-adjoint elliptic operators with a nonzero spectral flow. Such families exist (e.g., see [4]).

Denote the corresponding family of projections on the nonnegative spectral subspaces of the operators $A_{t}$ by $P_{t}$. From the definition of the spectral flow $\operatorname{sf} A_{t}$ as the net number of eigenvalues of the operators $A_{t}$ that pass through zero as the parameter $t$ varies, we obtain

$$
\operatorname{sf} A_{t}=d\left(P_{1}\right)-d\left(P_{0}\right) \neq 0 .
$$


The family $A_{t}$ is, however, periodic. Consequently, $P_{0}=P_{1}$ and $d\left(P_{1}\right)=d\left(P_{0}\right)$, which contradicts (四).

In the remaining part of the section we consider the class of even projections, where a similar functional can nevertheless be defined under the condition that the manifold $M$ where the projections act is odd-dimensional.

Definition 1 A pseudodifferential projection

$$
P: C^{\infty}(M, E) \longrightarrow C^{\infty}(M, E)
$$

is called even (cf. [B]) if its principal homogeneous symbol $\sigma(P)$ satisfies

$$
\sigma(P)(x, \xi)=\sigma(P)(x,-\xi) \quad \text { for all }(x, \xi) \in S^{*} M \text {. }
$$

The set of all even pseudodifferential projections of order zero is denoted by Even $(M)$. Let

$$
P^{*} M=S^{*} M / \mathbf{Z}_{2}
$$

be the bundle of projective spaces obtained as the quotient of the cotangent sphere bundle $S^{*} M$ under the action of the antipodal involution $\xi \rightarrow-\xi$. The corresponding projection is denoted by $\pi: S^{*} M \rightarrow P^{*} M$. Then the symmetry condition (5) means that the homogeneous principal symbol $\sigma(P)$ is the pullback of an endomorphism $\sigma^{\prime}(P)$ over the projective bundle $P^{*} M$ :

$$
\sigma(P)=\pi^{*} \sigma^{\prime}(P), \quad \sigma^{\prime}(P): \pi_{P}^{*} E \longrightarrow \pi_{P}^{*} E,
$$

where $\pi_{P}: P^{*} M \rightarrow M$ is the natural projection. Note that the Eq. (6) implies that an even projection determines a vector bundle on the projectivization $P^{*} M$,

$$
\operatorname{Im} \sigma^{\prime}(P) \in \operatorname{Vect}\left(P^{*} M\right) \text {. }
$$

Let $P_{1,2}$ be pseudodifferential projections

$$
P_{1,2}: C^{\infty}\left(M, E_{1,2}\right) \longrightarrow C^{\infty}\left(M, E_{1,2}\right)
$$

Their direct sum $P_{1} \oplus P_{2}$ is the projection

$$
P_{1} \oplus P_{2}=\left(\begin{array}{ll}
P_{1} & 0 \\
0 & P_{2}
\end{array}\right): C^{\infty}\left(M, E_{1} \oplus E_{2}\right) \longrightarrow C^{\infty}\left(M, E_{1} \oplus E_{2}\right) .
$$

Let us consider the following stable homotopy equivalence relation on the set Even $(M)$ of even projections. 
Definition 2 We say that two projections $P_{1,2} \in \operatorname{Even}(M)$ are equivalent and write $P_{1} \approx P_{2}$ if for some even projection $P: C^{\infty}(M, F) \longrightarrow C^{\infty}(M, F)$ there exists a homotopy of even projections

$$
P_{1} \oplus 0 \oplus P \sim 0 \oplus P_{2} \oplus P
$$

as projections in the ambient space $C^{\infty}\left(M, E_{1} \oplus E_{2} \oplus F\right)$.

Now consider the Grothendieck group generated by the semigroup Even $(M) / \approx$ consisting of classes of equivalent projections:

$$
K\left(P_{e v}(M)\right) \stackrel{\text { def }}{=} K(\operatorname{Even}(M) / \approx) .
$$

Each even projection $P$ defines an element in the group $K\left(P_{e v}(M)\right)$, which we denote by $[P]$. It is clear that the mapping $q: K\left(P_{e v}(M)\right) \longrightarrow K\left(P^{*} M\right)$ taking each even pseudodifferential projection to the range of its principal symbol is a homomorphism of abelian groups.

The above-mentioned properties of the rank of projections are formalized in the following definition.

Definition 3 A group homomorphism

$$
d: K\left(P_{e v}(M)\right) \longrightarrow \mathbf{R}
$$

is called a dimension if for any finite-dimensional projection $P$ we have

$$
d([P])=\operatorname{rank} P \equiv \operatorname{dim} \operatorname{Im} P .
$$

The following theorem describes all possible dimension functionals.

Theorem 1 The dimensions of even subspaces

$$
d: K\left(P_{e v}(M)\right) \longrightarrow \mathbf{R}
$$

are in a one-to-one correspondence with homomorphisms

$$
\chi: K(M) \longrightarrow \mathbf{R},
$$

which will be called "normalization homomorphisms." Moreover, for integer valued normalizations $\chi$ the dimension d takes rational values whose denominators can contain only powers of 2 :

$$
d: K\left(P_{e v}(M)\right) \longrightarrow \mathbf{Z}\left[\frac{1}{2}\right] .
$$


Proof. Consider the sequence

$$
0 \longrightarrow \mathbf{Z} \stackrel{i}{\longrightarrow} K\left(P_{e v}(M)\right) \stackrel{q}{\longrightarrow} K\left(P^{*} M\right) \longrightarrow 0
$$

where the map $i$ is induced by the homomorphism of semigroups

$$
\mathbf{Z}_{+} \rightarrow \operatorname{Even}(M) / \approx
$$

taking each nonnegative number $k$ to the class of a projection of rank $k$ acting, say, in the space $C^{\infty}(M)$. Let us prove that the sequence (9) is exact.

First, we verify that $q$ is an epimorphism. Indeed, an arbitrary vector bundle $\gamma \in$ Vect $\left(P^{*} M\right)$ can be realized as a subbundle in some trivial bundle,

$$
\gamma \subset \mathbf{C}^{N} \in \operatorname{Vect}\left(P^{*} M\right)
$$

By lifting this embedding to the cotangent spheres, we obtain

$$
\pi^{*} \gamma \subset \mathbf{C}^{N} \in \operatorname{Vect}\left(S^{*} M\right) \text {. }
$$

It is obvious that the orthogonal projection $\sigma=\sigma(x, \xi)$ on the subbundle $\pi^{*} \gamma$ is an even projection. Consider an arbitrary pseudodifferential projection $P$ with principal symbol $\sigma$. For the projection $P$ we obtain, by construction,

$$
q([P])=[\gamma] \in K\left(P^{*} M\right)
$$

This proves the exactness of the sequence in the third term.

The triviality of the composition $q \circ i$ is obvious. Let us check the inclusion $\operatorname{ker} q \subset \operatorname{Im} i$. Suppose that

$$
q\left([P]-\left[1_{N}\right]\right)=0
$$

where $P$ is an even projection in $C^{\infty}(M, E)$, and $1_{N}$ is the identity in $C^{\infty}\left(M, \mathbf{C}^{N}\right)$ (this does not restrict generality, since an arbitrary element of $K\left(P_{e v}(M)\right)$ is representable in this form). Condition (10) means that (possibly, after adding a trivial pair $\left(1_{N^{\prime}}, 1_{N^{\prime}}\right)$ to the pair $\left.\left(P, 1_{N}\right)\right)$ we obtain an isomorphism

$$
\operatorname{Im} \sigma^{\prime}(P) \simeq \mathbf{C}^{N}
$$

${ }^{2}$ Following [9], we can define $P$ by the formula

$$
P=-\frac{1}{2 \pi i} \int_{|\lambda-1|=\varepsilon}(\Pi-\lambda \mathrm{I})^{-1} d \lambda
$$

where $\Pi$ is an arbitrary pseudodifferential operator of order zero with principal symbol $\sigma$, and the number $\varepsilon, 0<\varepsilon<1$, is chosen in a way such that the circle $|\lambda-1|=\varepsilon$ contains no eigenvalues of $\Pi$. 
of vector bundles over $P^{*} M$. It follows that these bundles are homotopic as subbundles in the direct sum $\pi_{P}^{*} E \oplus \mathbf{C}^{N}$. Consider an arbitrary homotopy joining them and denote the corresponding family of projections by

$$
\begin{aligned}
\left\{\sigma_{t}^{\prime}\right\}_{t=0,1} & : \quad \sigma_{0}^{\prime}=\sigma^{\prime}(P) \oplus 0, \quad \sigma_{1}^{\prime}=0 \oplus 1_{N}, \\
\operatorname{Im} \sigma_{t}^{\prime} & \subset \quad \pi_{P}^{*} E \oplus \mathbf{C}^{N} \in \operatorname{Vect}\left(P^{*} M\right) \quad \text { for all } t \in[0,1] .
\end{aligned}
$$

The pullback of this homotopy to the cosphere bundle $S^{*} M$ will be denoted by $\sigma_{t}$. It follows from Statement 1 in [10] that there exists a (continuous) covering homotopy of pseudodifferential projections $P_{t}$,

$$
\sigma\left(P_{t}\right)=\sigma_{t},
$$

such that $P_{0}=P \oplus 0$ and $P_{1}$ differs from $0 \oplus 1_{N}$ by a compact operator. Accordingly, in the group $K\left(P_{e v}(M)\right)$ we obtain

$$
[P]-\left[1_{N}\right]=\left[P_{1}\right]-\left[0 \oplus 1_{N}\right] .
$$

It can be shown (e.g., see 11]) that projections differing by a compact operator are homotopic up to a finite rank projection. For example, in the case of a positive relative index of projections 5 ind $\left(P_{1}, 0 \oplus 1_{N}\right)=n \geq 0$ we obtain a homotopy of projections

$$
P_{1} \sim n \oplus 1_{N}
$$

where $n$ is a rank $n$ projection in $C^{\infty}(M, E)$. For negative ind $\left(P_{1}, 0 \oplus 1_{N}\right)=n<0$, we have

$$
P_{1} \sim 0 \oplus\left(1_{N}-(-n)\right),
$$

where $-n$, as before, is a projection of rank $-n>0$. In the first case we find that

$$
\left[P_{1}\right]-\left[0 \oplus 1_{N}\right]=[n]=i(n)
$$

in the Grothendieck group $K\left(P_{e v}(M)\right)$, and in the second case we also have

$$
\left[P_{1}\right]-\left[0 \oplus 1_{N}\right]=-[-n]=i(n)
$$

Let us finally verify the exactness of the sequence (9) in the first term. Suppose that for some $n>0$ we have

$$
i(n)=0 .
$$

\footnotetext{
${ }^{3}$ For projections $P, Q$ with compact difference, the relative index is defined as the index of the Fredholm operator $Q: \operatorname{Im} P \rightarrow \operatorname{Im} Q:$

$$
\text { ind }(P, Q) \stackrel{\text { def }}{=} \text { ind }(Q: \operatorname{Im} P \rightarrow \operatorname{Im} Q)=-\operatorname{ind}(P: \operatorname{Im} Q \rightarrow \operatorname{Im} P) .
$$
}


From the definition of the Grothendieck group it follows that for some even projection $P \in$ Even $(M)$ there exists a homotopy of even projections

$$
n \oplus P \sim 0 \oplus P \text {. }
$$

Moreover, without loss of generality, it can be assumed that the projection $P$ is the unit operator: $P=1_{N}$. Let us denote this continuous homotopy of even projections from $n \oplus P$ to $0 \oplus P$ by $P_{t}$. We obtain

$$
\text { ind }\left(P_{0}, P_{1}\right)=n \neq 0 \text {. }
$$

Let us show that the fact that the projections $P_{t}$ are even implies ind $\left(P_{0}, P_{1}\right)=0$. Indeed, without loss of generality it can be assumed that the family $\left\{P_{t}\right\}$ consists of orthogonal projections (the space of all projections can be linearly retracted to the space of orthogonal projections, th and the relative index (11) does not change under this retraction). Consider further an arbitrary periodic family $\left\{A_{t}\right\}_{t=0,1}$ of first-order self-adjoint pseudodifferential operators with homogeneous principal symbols on the cotangent spheres equal to $2 \sigma\left(P_{t}\right)-$ 1 (so that the positive spectral projection of the principal symbol of the operator $A_{t}$ coincides with $\left.\sigma\left(P_{t}\right)\right)$. The spectral flow of any periodic family $\left\{A_{t}\right\}_{t=0,1}$ can be expressed by the cohomological formula («4, cf. [12])

$$
\operatorname{sf}\left\{A_{t}\right\}_{t \in S^{1}}=\left\langle\operatorname{ch}\left(\sigma_{+}\left(A_{t}\right)\right) \pi^{*} \operatorname{Td}\left(T^{*} M \otimes \mathbf{C}\right),\left[S^{1} \times S^{*} M\right]\right\rangle, \quad \pi: S^{1} \times S^{*} M \rightarrow M .
$$

Here the vector bundle $\sigma_{+}\left(A_{t}\right) \in \operatorname{Vect}\left(S^{1} \times S^{*} M\right)$ is generated by the nonnegative spectral subspaces of the principal symbols $\sigma\left(A_{t}\right)$ (in our case $\sigma_{+}\left(A_{t}\right)=\operatorname{Im} \sigma\left(P_{t}\right)$ ), and $\mathrm{Td}$ is Todd class of a vector bundle.

On the oriented manifold $S^{*} M$ the involution $(x, \xi) \rightarrow(x,-\xi)$ reverses the orientation, while the cohomology class

$$
\operatorname{ch}\left(\sigma_{+}\left(A_{t}\right)\right) \pi^{*} \operatorname{Td}\left(T^{*} M \otimes \mathbf{C}\right)
$$

is invariant with respect to this involution. Thus, the spectral flow of the family $\left\{A_{t}\right\}_{t=0,1}$ is zero:

$$
\operatorname{sf}\left\{A_{t}\right\}_{t=0,1}=0 .
$$

Let us recall that the family of projections $P_{t}$ is a generalized spectral section [13] for the family $A_{t}$. Then, by virtue of one of the definitions of the spectral flow (see [10] or [13]), we obtain

$$
\text { sf }\left\{A_{t}\right\}_{t=0,1}=\text { ind }\left(P_{0}, P_{1}\right) .
$$

\footnotetext{
${ }^{4}$ The pseudodifferentiality of the orthogonal projection on the subspace $\operatorname{Im} P$ for an arbitrary pseudodifferential projection $P$ follows from the identity $\operatorname{Im} P=\operatorname{Im} P P^{*}$ and the self-adjointness of the operator $P P^{*}$.
} 
Hence,

$$
\text { ind }\left(P_{0}, P_{1}\right)=0 \text {, }
$$

which contradicts (11). The exactness of the sequence (9) is established.

Let us note that in terms of the sequence (9) the problem of describing dimension homomorphisms $d$ is reduced to the problem of closure of the following diagram to a commutative one:

$$
\begin{array}{lll}
\mathbf{Z} & \stackrel{i}{\rightarrow} & K\left(P_{e v}(M)\right) \\
\downarrow & \swarrow d & \\
\mathbf{R} & &
\end{array}
$$

(here $\mathbf{Z} \subset \mathbf{R}$ is the natural inclusion, and the map $d$ is so far unknown). First, note that the groups $K\left(P_{e v}(M)\right)$ and $K\left(P^{*} M\right)$ contain subgroups generated by bundles on the base $M$ :

$$
\begin{aligned}
& K(M) \rightarrow K\left(P^{*} M\right), \quad K(M) \stackrel{\alpha}{\rightarrow} K\left(P_{e v}(M)\right), \\
& {[E] \rightarrow\left[\pi_{P}^{*} E\right], \quad[E] \rightarrow\left[1_{C^{\infty}(M, E)}\right] .}
\end{aligned}
$$

Both maps are monomorphisms (this follows from the existence of a nonsingular vector field on an odd-dimensional manifold $M$ ), and moreover, these embeddings commute with $q$. Thus, there is a partial splitting of the sequence (9):

$$
0 \quad \rightarrow \quad \mathbf{Z} \oplus K(M) \stackrel{i \oplus \alpha}{\rightarrow} K\left(P_{e v}(M)\right) \stackrel{q}{\rightarrow} K\left(P^{*} M\right) / K(M) \rightarrow 0
$$

The quotient $K\left(P^{*} M\right) / K(M)$ is by [8] a purely torsion group (and the torsion is only in powers of 2). By virtue of the sequence (12), this implies that an arbitrary dimension

$$
d: K\left(P_{e v}(M)\right) \longrightarrow \mathbf{R}
$$

is uniquely determined by its restriction to the subgroup $K(M)$ (recall that on the first term in the sum $\mathbf{Z} \oplus K(M)$ the dimension $d$ is already defined, see Definition (3)):

$$
\chi: K(M) \longrightarrow \mathbf{R}
$$

This completes the proof of the theorem.

Remark 1 The dimension $d$ can be viewed as a generalization of the relative index of projections, since

$$
\text { ind }\left(P_{1}, P_{2}\right)=d\left(\left[P_{1}\right]-\left[P_{2}\right]\right)
$$

for two even projections $P_{1,2}$ with the same principal symbol.

For a more explicit expression for the dimension $d([P])$ with the trivial normalization $\chi \equiv 0$ see Sec. 6, where the relation with Gilkey's $\eta$-invariants is established. 


\section{Operators in subspaces. An index formula for even subspaces}

Consider two pseudodifferential projections

$$
P_{1,2}: C^{\infty}\left(M, E_{1,2}\right) \longrightarrow C^{\infty}\left(M, E_{1,2}\right)
$$

of order zero on a manifold $M$ and an $m$ th order pseudodifferential operator

$$
D: C^{\infty}\left(M, E_{1}\right) \longrightarrow C^{\infty}\left(M, E_{2}\right) .
$$

Suppose that $D$ acts in the subspaces determined by the projections, that is,

$$
D\left(\operatorname{Im} P_{1}\right) \subset \operatorname{Im} P_{2}, \quad P_{2} D P_{1}=D P_{1} .
$$

Then the restriction

$$
D: \operatorname{Im} P_{1} \longrightarrow \operatorname{Im} P_{2}
$$

is called an operator acting in subspaces. There is a criterion for the Fredholm property of operators of this form (see [9] and [2]). Before stating it, let us introduce the notion of the principal symbol of an operator in subspaces.

Definition 4 The principal symbol of the operator

$$
D: \operatorname{Im} P_{1} \longrightarrow \operatorname{Im} P_{2}
$$

acting in subspaces, is the homomorphism of vector bundles over $S^{*} M$ given by the restriction of the principal symbol of the operator $D$ :

$$
\sigma(D): \operatorname{Im} \sigma\left(P_{1}\right) \longrightarrow \operatorname{Im} \sigma\left(P_{2}\right) .
$$

A symbol is called elliptic if it is an isomorphism. In this case the operator $D$ is also called elliptic. In these terms, the following statement is valid; the proof is essentially contained in [2].

Proposition 1 An operator

$$
D: H^{s}\left(E_{1}\right) \supset \operatorname{Im} P_{1} \longrightarrow \operatorname{Im} P_{2} \subset H^{s-m}\left(E_{2}\right)
$$

is Fredholm if and only if it is elliptic.

The orders of the Sobolev spaces will be omitted for brevity in what follows.

Let us consider triples $\left(D, P_{1}, P_{2}\right)$ representing elliptic operators

$$
D: \operatorname{Im} P_{1} \longrightarrow \operatorname{Im} P_{2}
$$


such that $P_{1,2}$ are even projections in the sense of Definition 1 . These triples form a semigroup $\widetilde{L}_{e v}(M)$. Triples of the form $\left(1_{N}, 1_{N}, 1_{N}\right)$ are called trivial. Two triples $u_{1}$ and $u_{2}$ are called equivalent if for some trivial triple $u_{0}$ there exists a homotopy

$$
u_{1} \oplus u_{0} \sim u_{2} \oplus u_{0}
$$

We consider the Grothendieck group generated by the abelian semigroup of classes of equivalent triples:

$$
L_{e v}(M) \stackrel{\text { def }}{=} K\left(\widetilde{L}_{e v}(M) / \approx\right) .
$$

The index of a triple extends to a homomorphism of abelian groups

$$
\operatorname{ind}_{a}: L_{e v}(M) \longrightarrow \mathbf{Z} \text {. }
$$

Let us define the following two functionals on the group $L_{e v}(M)$ :

$$
\begin{aligned}
& \operatorname{ind}_{t}\left(D, P_{1}, P_{2}\right)=\operatorname{ind}_{t}\left(\sigma(D): \operatorname{Im} \sigma\left(P_{1}\right) \rightarrow \operatorname{Im} \sigma\left(P_{2}\right)\right) \\
& d\left(D, P_{1}, P_{2}\right)=d\left(P_{1}, P_{2}\right) .
\end{aligned}
$$

Consider the mapping

$$
\begin{aligned}
& d: L_{e v}(M) \longrightarrow \mathbf{Z}\left[\frac{1}{2}\right], \\
& d\left(D, P_{1}, P_{2}\right) \stackrel{\text { def }}{=} d\left(\left[P_{1}\right]-\left[P_{2}\right]\right) .
\end{aligned}
$$

It is defined by the projections $P_{1}, P_{2}$ and is independent of the operator acting between them. Moreover, the homomorphism (13) is independent of the normalization $\chi: K(M) \longrightarrow \mathbf{R}$. Let us verify the last statement. Without loss of generality, we can assume that the vector bundles $\operatorname{Im} \sigma^{\prime}\left(P_{1,2}\right)$ are pullbacks from the manifold $M$ :

$$
\operatorname{Im} \sigma^{\prime}\left(P_{1,2}\right)=\pi_{P}^{*} E_{1,2}
$$

Moreover, the vector bundles $E_{1,2}$ are isomorphic (an isomorphism is given by the elliptic symbol $\sigma(D)$ lowered to $M$ with the help of a nonsingular vector field on the manifold). Thus, the $K(M)$ components (in the sense of the sequence (12)) are equal for the two projections $P_{1,2}$.

Let us now construct a topological invariant of triples that extends to a homomorphism of groups

$$
\operatorname{ind}_{t}: L_{e v}(M) \longrightarrow \mathbf{Q} .
$$

This invariant is determined by the principal symbol of the operator alone. To this end, consider the quotient space

$$
\widetilde{P} M=\left\{S^{*} M \times[0,1] /(x, \pm \xi, 0)\right\} /(x, \pm \xi, 1)
$$




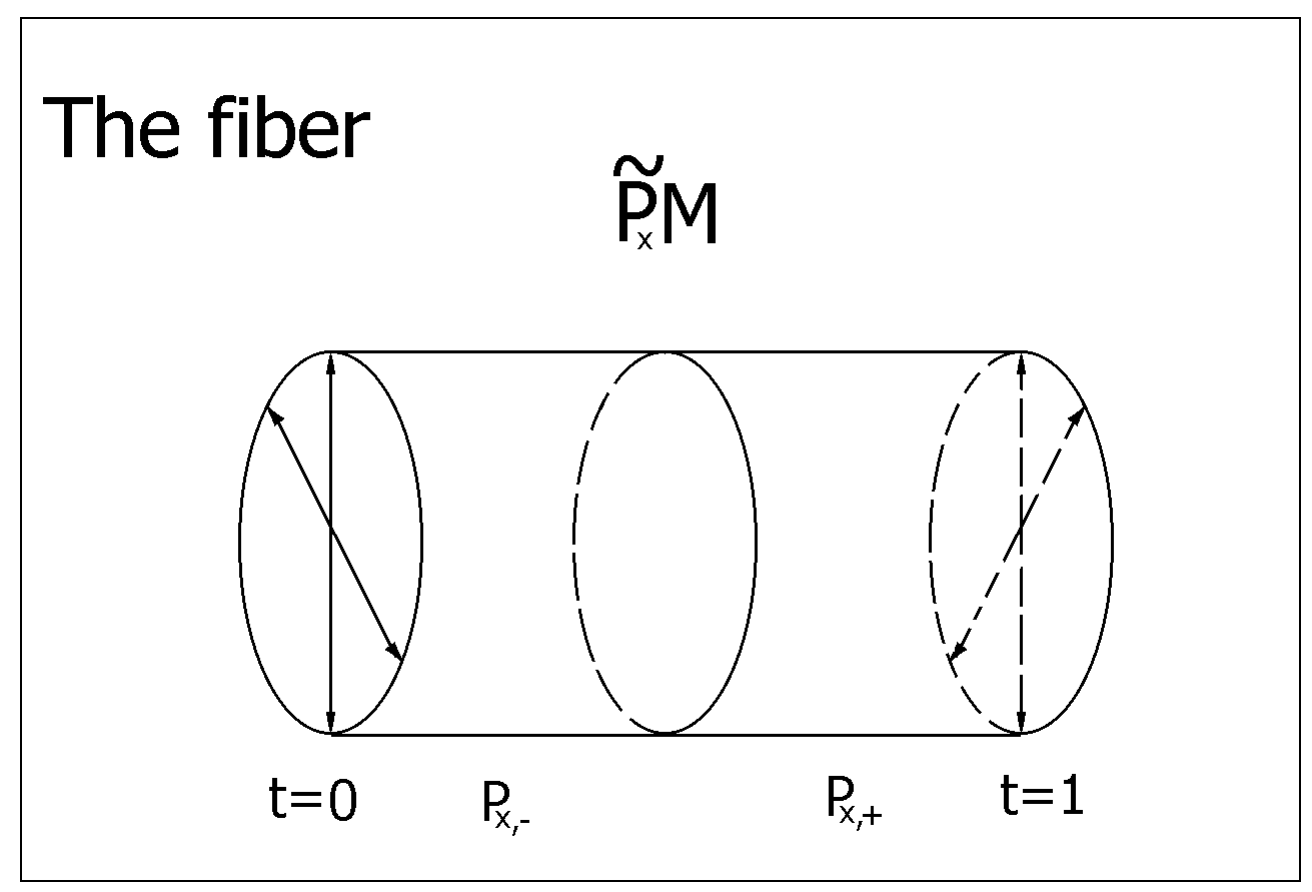

Figure 1:

(see Fig. 1).

This space is an oriented manifold with the structure of a fiber bundle over the manifold $M$. The fiber is formed by two odd-dimensional projective spaces with small disks deleted

$$
P_{-, x}=S_{x}^{*} M \times\left[0, \frac{1}{2}\right] /(x, \pm \xi, 0), P_{+, x}=S_{x}^{*} M \times\left[\frac{1}{2}, 1\right] /(x, \pm \xi, 1),
$$

glued along their common boundary $S_{x}^{*} M \times\left\{\frac{1}{2}\right\}$ (see Fig. 2):

$$
\widetilde{P} M=P_{-} M \bigcup_{S^{*} M} P_{+} M
$$

(the resulting fiber $\widetilde{P}_{x} M$ is an analog of the Klein bottle). Consider two natural projections in the bundles with unit closed interval as a fiber (see Fig. 2)

$$
P_{\mp} M \longrightarrow P^{*} M
$$

By means of these we can extend the vector bundles $\operatorname{Im} \sigma^{\prime}\left(P_{1,2}\right)$ from the space $P^{*} M \subset$ $P_{\mp} M$ to the entire $P_{\mp} M$, respectively. Then the isomorphism $\sigma(D)$ of these bundles over

\footnotetext{
${ }^{5}$ The projective space with a hole is a multidimensional analog of the Möbius band, but in our case it is orientable.
} 


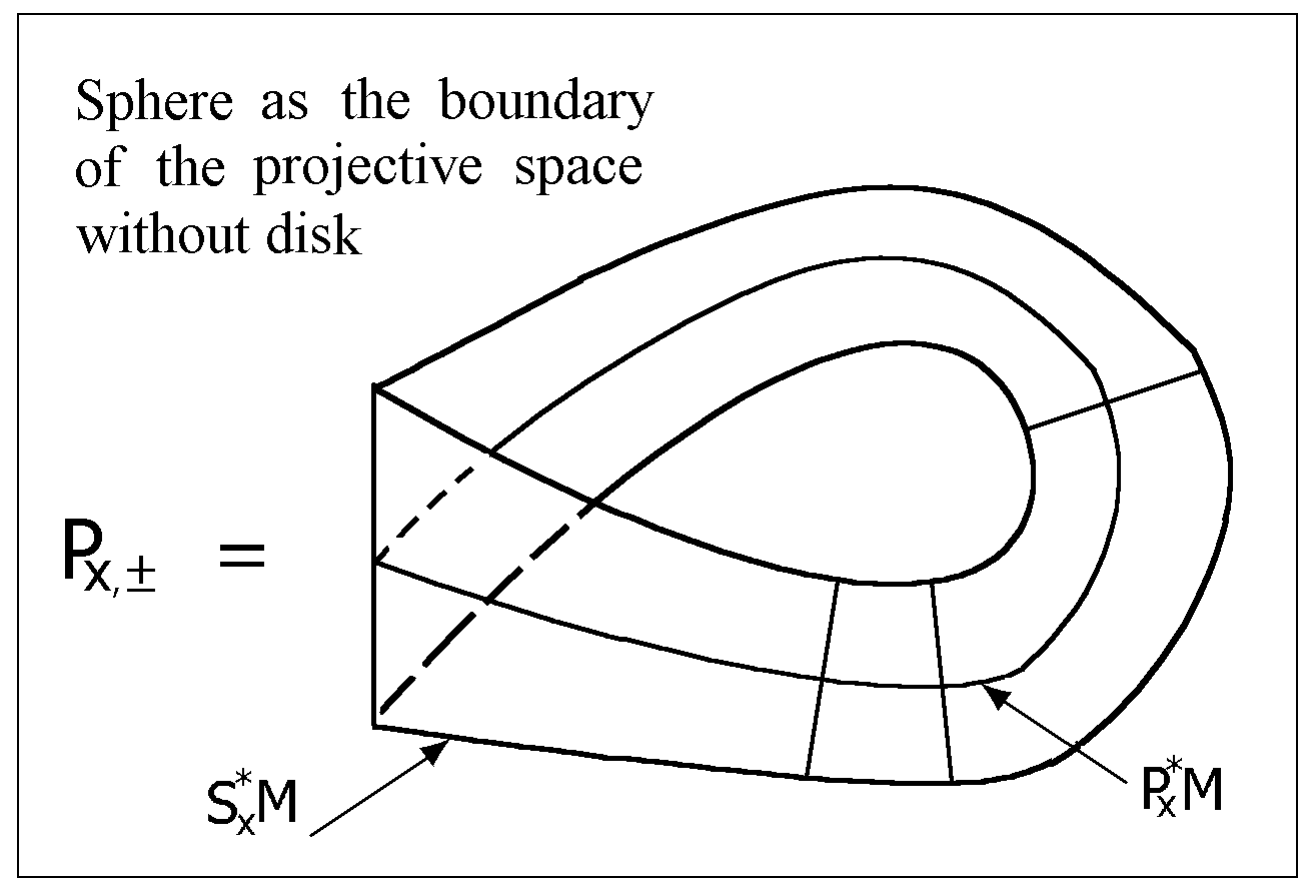

Figure 2:

the cotangent spheres $S^{*} M$ enables us to glue them; in this way we obtain a vector bundle over $\widetilde{P} M$ :

$$
\sigma\left(D, P_{1}, P_{2}\right) \in \operatorname{Vect}(\widetilde{P} M), \quad\left[\sigma\left(D, P_{1}, P_{2}\right)\right] \in K(\widetilde{P} M) .
$$

It is possible to define an analog of the usual "topological" index on the group $K(\widetilde{P} M)$ (cf. [14]):

$$
\operatorname{ind}_{t}\left(D, P_{1}, P_{2}\right)=\left\langle\operatorname{ch}\left[\sigma\left(D, P_{1}, P_{2}\right)\right] \operatorname{Td}\left(T^{*} M \otimes \mathbf{C}\right),[\widetilde{P} M]\right\rangle .
$$

In this notation we prove the following index formula, which is the main result of the present paper.

Theorem 2 Let $u=\left(D, P_{1}, P_{2}\right)$ be an elliptic operator acting in the subspaces defined by even pseudodifferential projections on an odd-dimensional manifold $M$. Then the following index formula is valid:

$$
\operatorname{ind}_{a} u=\operatorname{ind}_{t} u+d(u) \text {. }
$$

Proof. The left- and right-hand sides of (15) define homomorphisms of groups

$$
L_{e v}(M) \longrightarrow \mathbf{Q} \text {. }
$$

To prove the coincidence of these homomorphisms, by virtue of the absence of torsion in the group of rationals, it suffices to check the equality only on the elements of the 
group $L_{e v}(M)$ that rationally generate it. The exactness of the sequence (12) together with the fact that the group $K\left(P^{*} M\right) / K(M)$ is purely a torsion group imply that for an arbitrary triple $\left(D, P_{1}, P_{2}\right)$ the projections $P_{1,2}$ are rationally homotopic (i.e. a direct sum of the form $P_{1,2} \oplus \cdots \oplus P_{1,2}$ is homotopic) to projections that differ from projections on the spaces of sections of vector bundles by finite rank projections. Hence, as the elements rationally generating the group $L_{e v}(M)$, we can take triples of the form

$$
u=\left(\left(\begin{array}{cc}
0 & 0 \\
D & 0
\end{array}\right), 1_{E} \oplus n, m \oplus 1_{F}\right),
$$

where $E, F \in \operatorname{Vect}(M), n$ and $m$ are finite rank projections, and the operator

$$
D: C^{\infty}(M, E) \longrightarrow C^{\infty}(M, F)
$$

is a usual pseudodifferential operator. Let us show that formula (15) is true for operators of this form. Consider the relations

$$
\begin{aligned}
\operatorname{ind}_{a} u & =\operatorname{ind} D+n-m \\
d(u) & =n-m+d\left(\left[1_{C^{\infty}(M, E)}\right]-\left[1_{C^{\infty}(M, F)}\right]\right)=n-m
\end{aligned}
$$

(in the second one we use a nonsingular vector field to obtain an isomorphism of the bundles $E$ and $F$ ). Thus, to check formula (15) for a triple $u$, it suffices to verify that

$$
\operatorname{ind} D=\operatorname{ind}_{t}\left(D, 1_{C^{\infty}(M, E)}, 1_{C^{\infty}(M, F)}\right) .
$$

Let us show that the right-hand side of this formula is a slight modification of the usual Atiyah-Singer index formula for the operator $D$. We rewrite the Atiyah-Singer index formula in the form (see [14])

$$
\operatorname{ind} D=\left\langle\operatorname{ch}[\sigma(D)] \pi^{*} \operatorname{Td}\left(T^{*} M \otimes \mathbf{C}\right),[\widetilde{B} M]\right\rangle, \quad \widetilde{B} M=B_{-} M \bigcup_{S^{*} M} B_{+} M,
$$

where $B_{ \pm} M$ is the unit cotangent ball bundle for the manifold $M$. Next, consider the two expressions in (17) and (14) as integrals over the respective manifolds of characteristic classes represented via differential forms by means of connections in the corresponding vector bundles. The manifolds $\widetilde{B} M$ and $\widetilde{P} M$ are diffeomorphic in a neighborhood of $S^{*} M \times\left\{\frac{1}{2}\right\}$. We note also that these manifolds carry the orientation-reversing involution

$$
(x, \xi, t) \longrightarrow(x,-\xi, t)
$$

respecting their parts $P_{ \pm}$and $B_{ \pm}$. Consequently, for a connection in a vector bundle $\sigma\left(D, P_{1}, P_{2}\right)$ over $\widetilde{P} M$ that is invariant under the involution outside of a certain neighborhood of the cotangent spheres $S^{*} M \times\{1 / 2\}$, there is no contribution to the formula (16) 
from the corresponding domains (since the integrands are invariant under an orientationreversing involution); a similar cancellation happens on $\widetilde{P} M$. The remaining contribution, coming from the integration over a neighborhood of $S^{*} M \times\{1 / 2\}$, is the same for the two formulas, since the integrands coincide pointwise.

Thus, the index formula is proved for the case of projections with unit principal symbol. Such operators, as was noted above, rationally generate the whole group $L_{e v}(M)$. Hence, the index formula, as well as the theorem, is proved for the general case.

Let us make two important remarks concerning the topological term ind ${ }_{t}$ in the index formula.

Remark 2 In the definition of the group of stably homotopy equivalent triples $L_{e v}(M)$ we could have gone further by factorizing this group by the triples $(P, P, P)$ with an arbitrary even projection $P$, which obviously do not contribute to the index. Let us denote the resulting group by the same symbol $L_{e v}$. In this case we must replace the group $K(\widetilde{P} M)$ (where the principal symbol of the problem lies) by the quotient $K(\widetilde{P} M) / K\left(P^{*} M\right)$, and the corresponding principal symbol mapping becomes

$$
L_{e v}(M) \longrightarrow K(\widetilde{P} M) / K\left(P^{*} M\right) .
$$

The last group is an analog of the $K$-functor corresponding to the difference construction of the usual elliptic theory $K_{c}\left(T^{*} M\right)$ in view of the natural isomorphism

$$
K(\widetilde{P} M) / K\left(P^{*} M\right) \simeq K_{c}\left({ }^{p} T^{*} M\right), \text { where }{ }^{p} T^{*} M=P_{-} M \bigcup_{S^{*} M}\left\{T^{*} M \cap\{|\xi| \geq 1\}\right\} .
$$

The topological index

$$
K_{c}\left({ }^{p} T^{*} M\right) \longrightarrow \mathbf{Q}
$$

is given in this case by the same formula (14).

This statement in fact follows from the isomorphism (18), which, in turn, is geometrically obvious: the quotient group on the left-hand side is isomorphic to the relative group

$$
K(\widetilde{P} M) / K\left(P^{*} M\right) \simeq K\left(\widetilde{P} M, P_{+} M\right),
$$

and the noncompact spaces $\widetilde{P} M \backslash P_{+} M$ and ${ }^{p} T^{*} M$ are properly homeomorphic (i.e., there exists a homeomorphism given by a proper map).

Remark 3 The topological index ind ${ }_{t}$ of a triple can be reduced to the topological index of a usual elliptic operator. Namely, for a triple $\left(D, P_{1}, P_{2}\right)$ consider the symbol

$$
\sigma(D)^{-1}(x,-\xi) \sigma(D)(x, \xi) \oplus 1: \pi_{S}^{*} E_{1} \longrightarrow \pi_{S}^{*} E_{1}, \quad \pi_{S}: S^{*} M \rightarrow M,
$$


where the direct sum of symbols is taken with respect to the bundle decomposition

$$
\pi_{S}^{*} E=\operatorname{Im} \sigma\left(P_{1}\right)(x, \xi) \oplus \operatorname{Im}\left(1-\sigma\left(P_{1}\right)(x, \xi)\right) .
$$

For this symbol we have

$$
\operatorname{ind}_{t}\left(D, P_{1}, P_{2}\right)=\frac{1}{2} \operatorname{ind}_{t}\left(\sigma(D)^{-1}(x,-\xi) \sigma(D)(x, \xi) \oplus 1\right),
$$

where the right-hand side is the topological index of a usual elliptic operator in spaces.

To prove (19), we note that at the beginning of this section we could have taken this formula as a definition of $\operatorname{ind}_{t}\left(D, P_{1}, P_{2}\right)$. Furthermore, in the proof of the index theorem we would have to check that for a "classical" ' operator $D$ the expression on the right-hand side in (19) is equal to the (topological) index of $D$. Indeed, in this case we obtain

$$
\begin{gathered}
\operatorname{ind}_{t}\left(\sigma(D)^{-1}(x,-\xi) \sigma(D)(x, \xi) \oplus 1\right)=\operatorname{ind}_{t}(\sigma(D)-1(x,-\xi))+\operatorname{ind}_{t}(\sigma(D)(x, \xi))= \\
=-\operatorname{ind}_{t}(\sigma(D)(x,-\xi))+\operatorname{ind}_{t}(\sigma(D)(x, \xi))=2 \operatorname{ind}_{t}(\sigma(D)(x, \xi))=2 \operatorname{ind} D
\end{gathered}
$$

(in this chain of equalities only the symbols in the classical sense appear, and ind $\mathrm{d}_{t}$ is the usual topological index; in the next to the last equality we use the orientation-reversing involution $(x, \xi) \rightarrow(x,-\xi)$ on the manifold $S^{*} M$, and the last equality is the AtiyahSinger theorem).

\section{General boundary value problems}

Let us briefly recall the definition of general boundary value problems [2].

Let $M$ be a smooth compact manifold with boundary. Consider an $m$ th-order elliptic differential operator

$$
D: C^{\infty}(M, E) \rightarrow C^{\infty}(M, F)
$$

acting in the spaces of sections of vector bundles over $M$. For some collar neighborhood of the boundary with normal coordinate $t$ (the interior of the manifold corresponds to positive values of $t$ ), consider the jets of sections of the bundle $E$ in the normal direction:

$$
j_{\partial M}^{m-1} u=\left(u(0),\left.\frac{\partial}{\partial t} u\right|_{t=0}, \ldots,\left.\frac{\partial^{m-1}}{\partial t^{m-1}} u\right|_{t=0}\right): C^{\infty}(M, E) \rightarrow C^{\infty}\left(\partial M, E^{m}\right)
$$

here

$$
E^{m}=\underbrace{E \oplus E \oplus \ldots \oplus E}_{m \text { times }} .
$$

\footnotetext{
${ }^{6}$ That is, acting in spaces of sections on the base.
} 
A general boundary value problem is a system of equations of the form

$$
\begin{cases}D u=f, & u \in C^{\infty}(M, E), f \in C^{\infty}(M, F), \\ B j_{\partial M}^{m-1} u=g \in \operatorname{Im} P, & \operatorname{Im} P \subset C^{\infty}(\partial M, G) .\end{cases}
$$

Here the pseudodifferential operator $P$

$$
P: C^{\infty}(\partial M, G) \rightarrow C^{\infty}(\partial M, G)
$$

is a zero-order projection, $P^{2}=P$, and the boundary operator $B$

$$
B: C^{\infty}\left(\partial M, E^{m}\right) \rightarrow C^{\infty}(\partial M, G)
$$

is also a pseudodifferential operator whose range is contained in the subspace $\operatorname{Im} P: P B=$ $B$. The boundary value problem (20) is denoted by $(D, B, P)$. The classical boundary value problems correspond to the special case of the unit projection $P=1$ in formula (20).

To state the Fredholm criterion for these boundary value problems, for each point $\left(x, \xi^{\prime}\right)$ of the cosphere bundle $S^{*} \partial M$ of the boundary we consider the following ordinary differential equation with constant coefficients on the line $\mathbf{R} \ni t$ :

$$
\sigma(D)\left(x, 0,-i \frac{d}{d t}, \xi^{\prime}\right) u(t)=0
$$

(here $\sigma(D)$ is the principal symbol of $D$ ). Let us denote the subspace of Cauchy data of solutions that are bounded as $t \rightarrow \pm \infty$ by $L^{\mp}\left(x, \xi^{\prime}\right)$. Then for an elliptic differential operator $D$ one has the decomposition

$$
E_{x}^{m}=L^{+}\left(x, \xi^{\prime}\right) \bigoplus L^{-}\left(x, \xi^{\prime}\right) .
$$

Moreover, in this case the families $L^{ \pm}\left(x, \xi^{\prime}\right)$ define smooth vector subbundles

$$
L^{ \pm} \subset \pi^{*} E^{m}, \quad \text { where } \quad \pi: S^{*} \partial M \rightarrow \partial M .
$$

Remark 4 It can be shown that the decomposition (21) is a necessary and sufficient condition for the ellipticity of the operator $D$ on the boundary $\partial M$.

The following criterion for the Fredholm property of general boundary value problems [2] holds.

Theorem 3 The boundary value problem $(D, B, P)$ defines a Fredholm operator

$$
\left(\begin{array}{c}
D \\
B j_{\partial M}^{m-1}
\end{array}\right): H^{s}(M, E) \rightarrow \begin{gathered}
H^{s-m}(M, F) \\
\bigoplus_{\operatorname{Im} P \subset H^{\delta}}(\partial M, G)
\end{gathered}
$$


for $s>m-1 / 2$ if and only if the operator $D$ is elliptic and the restriction of the principal symbol of the boundary operator $B$ to the subbundle $L^{-}$defines an isomorphism

$$
\sigma(B): L^{-}(D) \rightarrow \operatorname{Im} \sigma(P) .
$$

It is supposed here that the orders of the components of the operator $B$ with respect to the jet $j_{\partial M}^{m-1}$ are consistent with the indices $s, \delta$ of Sobolev spaces.

The class of boundary value problems for differential operators is too narrow for making homotopies of elliptic symbols. It turns out that the following simple generalization of this class enables us to carry out the necessary homotopies; hence, we can apply topological methods to the index problem for the general boundary value problems.

In this paper we will use operators which are differential with respect to the normal variable $t$. Namely, let us consider operators $D$ on the manifold $M$ that have the form

$$
D=\sum_{k=0}^{m} A_{k}(t)\left(-i \frac{\partial}{\partial t}\right)^{k}
$$

in a collar neighborhood of the boundary, where $A_{k}(t)$ are smooth families of pseudodifferential operators of orders $m-k$, the operator $A_{m}(t)$ being a homomorphism of vector bundles. Second, on the entire manifold the operator $D$ must be represented by the construction of a pseudodifferential operator with a continuous symbol (see [15], [16] or [6]). Let us note that the necessity to consider operators with continuous symbols stems from the fact that the symbol of the operator (22) already is not smooth in general. $\overline{7}$

For the operators of the class just defined, the general boundary value problems are posed in just the same way as in the usual classical case (20). The definitions of the subspaces $L^{ \pm}$and the criterion for the Fredholm property remain valid (Theorem 3).

Let us consider examples of boundary value problems for operators of the form (22). These special boundary value problems will enable us (in the classical case) to reduce the index problem to the known case of a closed manifold.

Example 1 Let a vector bundle $E$ in a neighborhood of the boundary $\partial M$ be decomposed into a sum of two subbundles

$$
\left.E\right|_{U_{\partial M}}=E_{+} \oplus E_{-} .
$$

For the two bundles $\left.E_{ \pm}\right|_{\partial M}$ consider first-order elliptic operators $\Lambda_{ \pm}$with the principal symbols equal to $\left|\xi^{\prime}\right|$. We also choose a first-order operator $\Lambda$ with principal symbol $|\xi|$ acting in the bundle $E$ on the entire manifold $M$. Let us define, in conformity with the decomposition (23), the following elliptic first order operator in a collar neighborhood of the boundary:

$$
D=\left(-i \frac{\partial}{\partial t}+i \Lambda_{+}\right) \oplus\left(+i \frac{\partial}{\partial t}+i \Lambda_{-}\right): C^{\infty}\left(U_{\partial M}, E\right) \rightarrow C^{\infty}\left(U_{\partial M}, E\right) .
$$

7 This is the well-known problem of nonpseudodifferentiality of tensor products (see [15]). Here it is, for example, the composition of operators $\frac{\partial}{\partial t}$ and $A_{1}(t)$. 
For the principal symbol of this operator on the boundary we obtain the equality

$$
L^{-}(D)=0 \oplus \pi^{*} E_{-} .
$$

Hence, the following boundary condition for this operator is elliptic:

$$
\left.u_{-}\right|_{\partial M}=g \in C^{\infty}\left(\partial M, E_{-}\right), \quad \text { for } u=\left(u_{+}, u_{-}\right) \in C^{\infty}\left(U_{\partial M}, E_{+} \oplus E_{-}\right) .
$$

This operator can be extended to the entire manifold. To this end, consider a smooth cutoff function $\chi$ on $M, 0 \leq \chi(t) \leq 1$, which is identically equal to 1 for $0 \leq t \leq 1 / 3$ and vanishes for $t \geq 2 / 3$. The desired extension is now given, say, by the formula

$$
D=\chi(t)\left[\left(-i \frac{\partial}{\partial t}+i \Lambda_{+}\right) \oplus\left(+i \frac{\partial}{\partial t}+i \Lambda_{-}\right)\right]+(1-\chi(t)) i \Lambda .
$$

The operator $D$ with the boundary condition (25) defines an elliptic boundary value problem. It is well known that this boundary value problem has index zero. This fact can be proved by noting that the family of boundary value problems

$$
D+i p
$$

is an elliptic family with parameter $p$ in the half-plane Rep $>0$ in the sense of AgranovichVishik [17]. Consequently, it is invertible for large values of $p$. Besides, the invertibility of the family $D+i p$ can be shown directly (see [6]). Correcting the operator $D$ by a finite-dimensional operator, we can suppose that $D$ is invertible itself.

In the next section we carry out reductions of boundary value problems, and there it will be more convenient not to consider an explicit homotopy of the operator $D$ on the manifold $M$, but rather consider a homotopy of its restriction to a small neighborhood of the boundary. More precisely, we start from homotopies of the form

$$
\sum_{k=0}^{m} A_{k}(t, \tau)\left(-i \frac{\partial}{\partial t}\right)^{k}
$$

defined for small values of the parameter $t$, for example, for $t<1$. Here $\tau \in[0,1]$ is the parameter of the homotopy. It is easy to note that for such a homotopy in a neighborhood of the boundary, one can construct a homotopy of the operator $D$ on the entire manifold. The required homotopy of the operator $D$ for $t \geq 1$ is constant, while in the remaining part of the collar neighborhood of the boundary it is equal to (for the cutoff function $\chi(t)$ from the example discussed above)

$$
D_{\tau}=\sum_{k=0}^{m} A_{k}(t, \tau \chi(t))\left(-i \frac{\partial}{\partial t}\right)^{k} .
$$




\section{From general to spectral boundary value problems}

In the present section we show that the methods of index theory of classical boundary value problems [6] enable us to reduce a general boundary value problem to a certain spectral boundary value problem in a canonical way. Moreover, the reduction process does not affect the space of boundary data defined by the projection $P$. We divide the reduction procedure into several stages.

Let us briefly comment on the corresponding constructions. Steps 1 and 2 of the reduction are auxiliary in the sense that here the boundary operator $B$ does not change. On the third step, which is the basis of the construction, we produce a homotopy of the boundary operator $B$ to the trivial one.

\section{Step 1. Reduction to first-order operators}

The index problem for the boundary value problem $(D, B, P)$ for an operator of order $m$ is reduced to a similar problem for a first-order operator by the following theorem.

Theorem 4 An elliptic boundary value problem $(D, B, P), \operatorname{deg} D=m$, is stably homotopic to the boundary value problem

$$
\left(D^{\prime}, B^{\prime}, P\right) \circ m D_{+}^{m-1}, \quad m D_{+}^{m-1} \stackrel{\text { def }}{=} \underbrace{D_{+}^{m-1} \oplus \ldots \oplus D_{+}^{m-1}}_{m \text { times }}
$$

where $D_{+}$is the invertible first-order operator from Example 1 (for the vector bundles $\left.E_{-}=0, E=E_{+}\right)$that does not require boundary conditions and $\left(D^{\prime}, B^{\prime}, P\right)$ is a boundary value problem for a first order operator. In addition, the projection $P$ is constant in the homotopy.

Proof. Consider the direct sum of boundary value problems

$$
(D, B, P) \oplus \bigoplus_{1}^{m-1} D_{+}^{m}
$$

Its index coincides with the index of the original problem, since the operator $D_{+}$is invertible. Let us represent the operator $D$ in the form

$$
D=\sum_{k=0}^{m} D_{k}(t)\left(-i \frac{\partial}{\partial t}-i \Lambda\right)^{k}\left(-i \frac{\partial}{\partial t}+i \Lambda\right)^{m-k} \equiv \sum_{k=0}^{m} D_{k}(t) D_{-}^{k} D_{+}^{m-k}
$$

where $\Lambda$ is an operator with principal symbol $\left|\xi^{\prime}\right|$ acting in the vector bundle $E$ in a neighborhood of the boundary. Consider the following homotopy of operators (in these 
matrices, we omit the argument $t$ of the coefficients $D_{k}(t)$ for brevity)

$$
\begin{aligned}
D_{\tau}= & \left(\begin{array}{cccc}
D-\tau^{m} D & 0 & \ldots & 0 \\
0 & D_{+}^{m} & \ldots & 0 \\
0 & 0 & D_{+}^{m} & \ldots \\
0 & 0 & \ldots & D_{+}^{m}
\end{array}\right)+ \\
& \left(\begin{array}{cccc}
\tau^{m} D_{0} D_{+}^{m} & \tau^{m-1} D_{1} D_{+}^{m} & \ldots & \tau D_{m-1} D_{+}^{m}+\tau D_{m} D_{-} D_{+}^{m-1} \\
-\tau D_{-} D_{+}^{m-1} & 0 & \ldots & 0 \\
0 & -\tau D_{-} D_{+}^{m-1} & \ldots & \ldots \\
0 & 0 & \ldots & 0
\end{array}\right) .
\end{aligned}
$$

At the initial point $\tau=0$ we have $D_{\tau=0}=D \oplus \bigoplus_{1}^{m-1} D_{+}^{m}$. On the other hand, for $\tau=1$ the factorization required in the theorem is obtained:

$$
D_{\tau=1}=\left(\begin{array}{cccc}
D_{0}(t) D_{+} & D_{1}(t) D_{+} & \ldots & D_{m-1}(t) D_{+}+D_{m}(t) D_{-} \\
-D_{-} & D_{+} & 0 & 0 \\
0 & -D_{-} & D_{+} & \ldots \\
0 & 0 & \ldots & D_{+}
\end{array}\right) \circ m D_{+}^{m-1} .
$$

Let us verify that the operator $D_{\tau}$ is elliptic for $\tau \in[0,1]$. First of all, we calculate the subspace $L^{-}\left(D_{\tau}\right)$. Let $U=\left(U_{0}(t), U_{1}(t), \ldots, U_{m-1}(t)\right)$ be a bounded solution as $t \rightarrow+\infty$ of the equation

$$
\sigma\left(D_{\tau}\right)\left(x, 0,-i \frac{d}{d t}, \xi^{\prime}\right) U=0
$$

We would like to note that the derivatives of the solution $U$ are bounded too, since it is a solution of an ordinary differential equation (28) with constant coefficients. Writing out this equation componentwise, we obtain the system of equations

$$
\left\{\begin{array}{l}
\left(1-\tau^{m}\right) \sigma(D)\left(-i \frac{d}{d t}\right) U_{0}+\left(-i \frac{d}{d t}+i\right)^{m}\left(d_{0} \tau^{m} U_{0}+\ldots+d_{m-1} \tau U_{m-1}\right)+ \\
+\tau\left(-i \frac{d}{d t}+i\right)^{m-1}\left(-i \frac{d}{d t}-i\right) d_{m} U_{m-1}=0, \\
\left(-i \frac{d}{d t}+i\right)^{m} U_{j}=\tau\left(-i \frac{d}{d t}+i\right)^{m-1}\left(-i \frac{d}{d t}-i\right) U_{j-1} \quad \text { for } 0<j<m
\end{array}\right.
$$

(here by $d_{j}$ we denote the principal symbols of the operators $D_{j}(0)$ ). Since the equation

$$
\left(-i \frac{d}{d t}+i\right) u=0
$$

has no bounded solutions on the half-line, we can cancel this operator in the system (29) in all equations except the first one. Hence, we obtain

$$
\left(-i \frac{d}{d t}+i\right) U_{j}=\tau\left(-i \frac{d}{d t}-i\right) U_{j-1} .
$$


Successively substituting these relations into one another, we obtain

$$
\left(-i \frac{d}{d t}+i\right)^{j} U_{j}=\tau^{j}\left(-i \frac{d}{d t}-i\right)^{j} U_{0}
$$

Taking into account these equations in the first equation in (29), we find that it is reduced to the requirement

$$
\sigma(D)\left(-i \frac{d}{d t}\right) U_{0}=0
$$

This implies the ellipticity of the operator $D_{\tau}$ : equation (30) on the entire line has no bounded solutions, i.e. the decomposition (21) is valid.

Thus, the following description of the subbundle $L^{-}\left(D_{\tau}\right)$ is obtained. The projection on the first term in the sum

$$
E \oplus \bigoplus_{1}^{m-1} E \stackrel{p r}{\longrightarrow} E
$$

induces an isomorphism of vector bundles

$$
L^{-}\left(D_{\tau}\right) \stackrel{p r}{\longrightarrow} L^{-}(D)
$$

the preimage of an element $u \in L^{-}(D)$ under this map is given by the formula

$$
\begin{aligned}
U & =\left(U_{0}, \ldots, U_{m-1}\right) \\
U_{0} & =u \\
\left(-i \frac{d}{d t}+i\right)^{j} U_{j} & =\tau^{j}\left(-i \frac{d}{d t}-i\right)^{j} U_{0} .
\end{aligned}
$$

Let us decompose the boundary operator in the same way as the operator $D$ has been rewritten in the above:

$$
B j_{\partial M}^{m-1}=\left.\sum_{k=0}^{m-1} B_{k}\left(-i \frac{\partial}{\partial t}-i \Lambda\right)^{k}\left(-i \frac{\partial}{\partial t}+i \Lambda\right)^{m-1-k}\right|_{t=0}
$$

Therefore, the principal symbol of the boundary condition

$$
B j_{\partial M}^{m-1} \circ p r: C^{\infty}\left(M, E \oplus \bigoplus_{1}^{m-1} E\right) \rightarrow C^{\infty}(\partial M, G)
$$

on the subspace $L^{-}\left(D_{\tau=1}\right)$ has the factorization (by virtue of (31))

$$
\sigma\left(B^{\prime}\right) j_{\partial M} \circ\left(-i \frac{d}{d t}+i\right)^{m-1}, \quad \text { where } \quad\left(\sigma\left(B^{\prime}\right) j_{\partial M}\right) U=\sum_{k=0}^{m-1} b_{k} U_{k}(0) .
$$


Hence, the homotopy

$$
\left(D_{\tau}, B \circ p r, P\right), \quad \tau \in[0,1],
$$

of elliptic boundary value problems connects the initial problem (27) with the boundary value problem that is equal to the composition indicated in the theorem:

$$
\left(D_{\tau=1}, B \circ p r, P\right)=\left(D^{\prime}, B^{\prime}, P\right) \circ m D_{+}^{m-1} .
$$

This completes the proof of the theorem.

\section{Step 2. An expression for the operator on the boundary via the Calderón projection}

At this step we show that a first order operator is reduced in a collar neighborhood of the boundary to an operator that is uniquely determined by the subbundle $L^{-}(D)$.

Consider a boundary value problem for the first-order elliptic operator

$$
D=-i A_{1} \frac{\partial}{\partial t}+A_{0}: C^{\infty}(M, E) \rightarrow C^{\infty}(M, F) .
$$

The coefficient $A_{1}$ is by assumption an isomorphism of bundles in a neighborhood of the boundary. With the help of this isomorphism, we identify the bundles $E$ and $F$. Then the operator $D$ becomes

$$
D=-i \frac{\partial}{\partial t}+A
$$

The ellipticity of $D$ implies that the eigenvalues of the principal symbol of the operator $A$ for nonzero values of the cotangent variable $\xi^{\prime}$ on the boundary have nonzero imaginary parts. A simple calculation shows that the subbundle $L^{-}(D)$ is generated by the spectral subspaces of the symbol $\sigma(A)$ with negative imaginary parts of the eigenvalues. Let us denote the corresponding spectral projection by $q$ :

$$
q: \pi^{*} E \rightarrow \pi^{*} E, \quad \operatorname{Im} q=L^{-}(D) .
$$

Let us also consider an arbitrary pseudodifferential operator $Q$ (not necessarily a projection) with principal symbol $q$. Such operators are called Calderón projections for the operator $D$ (see [6]).

Consider a linear homotopy of operators

$$
D_{\tau}=-i \frac{\partial}{\partial t}+(1-\tau) A-i \tau(2 Q-1) \Lambda
$$

where $\Lambda$ is an operator with principal symbol equal to $\left|\xi^{\prime}\right|$. Since the projection $q\left(\xi^{\prime}\right)$ is the spectral projection for the symbol $\sigma(A)\left(\xi^{\prime}\right)$, we see that the eigenvalues of the symbol

$$
(1-\tau) \sigma(A)\left(\xi^{\prime}\right)-i \tau(2 q-1)\left|\xi^{\prime}\right|
$$


are, respectively, equal to (here $\lambda$ is an arbitrary eigenvalue of the symbol $\sigma(A)$ )

$$
(1-\tau) \lambda+i \tau \operatorname{sign}(\operatorname{Im} \lambda)
$$

This implies the ellipticity of the operator $D_{\tau}$ and the independence of the subbundle $L^{-}\left(D_{\tau}\right)$ of the parameter $\tau$.

In this way, we present a homotopy of boundary value problems

$$
\left(D_{\tau}, B, P\right), \quad \tau \in[0,1] .
$$

As a result, the principal symbol of the operator $D$ obtained is determined on the boundary by the principal symbol of the Calderón projection $Q$

$$
D_{\tau=1}=-i \frac{\partial}{\partial t}-i(2 Q-1) \Lambda
$$

At the end of this step, let us consider the orthogonal projection $q^{\prime}$ onto the subbundle $L^{-}(D)$ and a pseudodifferential projection $Q^{\prime}$ with principal symbol $q^{\prime}$. Then a linear homotopy

$$
-i \frac{\partial}{\partial t}-i\left(2\left(\tau Q^{\prime}+(1-\tau) Q\right)-1\right) \Lambda
$$

leads for $\tau=1$ to an operator

$$
-i \frac{\partial}{\partial t}-i\left(2 Q^{\prime}-1\right) \Lambda
$$

with principal symbol uniquely determined by the subbundle $L^{-}(D)$. Hence, it can be assumed in what follows that the symbol $q$ of the Calderón projection is the orthogonal projection onto the subbundle $L^{-}(D)$.

\section{Step 3. Reduction of the Calderón projection to the projection of the boundary data}

In this section we make a homotopy of the operators $D$ and $B$ of the boundary value problem $(D, B, P)$. As a result of this homotopy, the boundary operator $B$ is transformed from an operator with principal symbol giving an isomorphism of subbundles

$$
L^{-}(D) \stackrel{\sigma(B)}{\longrightarrow} \operatorname{Im} \sigma(P)
$$

to the identity operator. The bundle $L^{-}(D)$, in particular, is deformed into $\operatorname{Im} \sigma(P)$. Formula (32) shows that to construct such a homotopy of boundary value problems, it suffices to produce a homotopy of the principal symbol of the Calderón projection $q$, as well as of the principal symbol of the boundary operator $\sigma(B)$. From the theory of vector bundles it is known that isomorphic subbundles (in our case these are $L^{-}(D)$ and $\operatorname{Im} \sigma(P)$ ) 
can be deformed into one another as subbundles in the ambient vector bundle (provided the dimension of this bundle is large enough). In this particular situation, let us write down an explicit formula for such a homotopy.

Let us realize the bundle $G \in \operatorname{Vect}(\partial M)$ as a subbundle of the trivial $\mathbf{C}^{N}$

$$
G \stackrel{\alpha}{\complement} \mathbf{C}^{N} \text {. }
$$

Recall that the boundary data of the boundary value problem lie in the space of sections of this bundle: $\operatorname{Im} P \subset C^{\infty}(\partial M, G)$.

Consider the direct sum of boundary value problems

$$
(D, B, P) \oplus D_{+},
$$

where an invertible operator $D_{+}$is the operator from Example 1 corresponding to the bundles $E_{+}=E=\mathbf{C}^{N}$. The desired homotopy of subbundles

$$
L^{-}(D) \oplus 0,0 \oplus \operatorname{Im} \sigma(P) \subset \pi^{*}\left(E \oplus \mathbf{C}^{N}\right)
$$

in this notation is a homotopy of rotation by $90^{\circ}$ with the help of the symbol $\sigma(B)$. More precisely, for an angle $\tau \in[0, \pi / 2]$ we define the subbundle

$$
L^{-}(\tau) \subset \pi^{*}\left(E \oplus \mathbf{C}^{N}\right)
$$

whose fiber over a point $\left(x, \xi^{\prime}\right) \in S^{*} \partial M$ is generated by vectors of the form

$$
v_{\tau}=(\cos \tau v, \sin \tau \sigma(B) v) \quad \text { for all } v \in L^{-}(D) .
$$

The homotopy of the principal symbol of the boundary operator $B$ is carried out by the formula

$$
\sigma\left(B_{\tau}\right) v_{\tau} \stackrel{\text { def }}{=} \sigma(B) v
$$

It is clear from (33) that at the end of the homotopy for $\tau=\pi / 2$ we obtain

$$
L^{-}(\pi / 2)=0 \oplus \operatorname{Im} \sigma(P), \quad \sigma\left(B_{\pi / 2}\right)=\operatorname{Id} .
$$

Thus, the operator $\left(D_{\pi / 2}, B_{\pi / 2}, P\right)$ defines the desired spectral boundary value problem.

This completes the reduction of a general boundary value problem to the corresponding spectral boundary value problem of the form (2).

\section{An index formula for even boundary value prob- lems}

In this section we obtain an index formula for spectral boundary value problems of the form (2) under the additional assumption that the projection $P$ is even.

\footnotetext{
${ }^{8}$ In combination with the reductions of the previous section, this implies an index formula for boundary value problems of the general form (11) under the parity condition.
} 
index formula by a reduction to a classical boundary value problem. To this end, let us study the relationship between classical and even boundary value problems.

Consider the Grothendieck group $K\left(P_{e v}(X)\right)$ generated by the abelian semigroup of classes of equivalent even projections (see formula (8)). It has the subgroup generated by unit projections. The corresponding quotient group is denoted by $K\left(P_{e v}(X)\right) / K(X)$.

A spectral boundary value problem of the form (2) on the manifold $M$ is denoted by $\mathcal{D}=(D, P)$. Such a boundary value problem is called even if the projection $P$ is an even projection on the boundary $\partial M$. Let us introduce an equivalence relation for even boundary value problems. Namely, two boundary value problems $\mathcal{D}_{1}$ and $\mathcal{D}_{2}$ are called equivalent if there exists an even homotopy

$$
\mathcal{D}_{1} \oplus D_{+} \sim D_{+} \oplus \mathcal{D}_{2}
$$

where $D_{+}$is, as in the previous section, an invertible operator from the Example 1 for the choice of bundles $E=E_{+}$. The Grothendieck group of even boundary value problems is denoted by $K\left(\mathcal{D}_{e v}(M)\right)$. It has the subgroup generated by the classical boundary value problems from Example 1 for arbitrary vector bundles $E$ on $M$ and $E_{+}$on $\partial M$, respectively. The quotient group is denoted by

$$
K\left(\mathcal{D}_{e v}(M)\right) /(K(\partial M) \oplus K(M)) .
$$

Finally, we need the group

$$
K(\mathcal{D}(M)) /(K(\partial M) \oplus K(M))
$$

generated by classical boundary value problems $(P=1)$. It is obtained by the same construction as before, with the replacement of the condition that the projection $P$ is even by the condition $P=1$. The resulting quotient group, which is similar to (34), does not require a new notation, since it can be identified with the usual $K$-group of vector bundles with compact support

$$
K(\mathcal{D}(M)) /(K(\partial M) \oplus K(M)) \stackrel{\gamma}{\rightarrow} K_{c}\left(T^{*}(M \backslash \partial M)\right) \equiv K_{c}\left(T^{*} \stackrel{\circ}{M}\right) .
$$

Let us recall the definition of the map $\gamma$. First, we use the isomorphisms

$$
K_{c}\left(T^{*}(M \backslash \partial M)\right) \simeq K\left(B^{*} M, \partial\left(B^{*} M\right)\right), \quad \partial\left(B^{*} M\right)=\left.S^{*} M \cup B^{*} M\right|_{\partial M},
$$

where $B^{*} M$ is the unit ball bundle of the manifold $M$, and $\partial\left(B^{*} M\right)$ is its full boundary. This allows us to identify the elements of the group $K_{c}\left(T^{*} \stackrel{\circ}{M}\right)$ with the help of the difference construction both with isomorphisms of vector bundles $E, F \in \operatorname{Vect}(M)$

$$
\sigma: \pi^{*} E \rightarrow \pi^{*} F, \quad \pi: S^{*} M \rightarrow M
$$


that do not depend on $\xi$ in a neighborhood of the boundary and with isomorphisms defined everywhere on $\partial\left(B^{*} M\right)$.

Consider a classical boundary value problem $(D, 1)$ of the form (21). In a neighborhood of the boundary it can be obviously rewritten as the boundary value problem from Example 1. Then the homomorphism $\gamma$ is by definition equal to

$$
\gamma(D, 1) \stackrel{\text { def }}{=} \sigma(D)(\xi)+i \chi(t)
$$

for a cutoff function $\chi(t)$ equal to 1 on $\partial M$, as above. It follows from (24) that the principal symbol (37) is invertible on $\partial\left(B^{*} M\right)$. It is also not difficult to construct the inverse mapping for $\gamma$ :

$$
K_{c}\left(T^{*} \stackrel{\circ}{M}\right) \stackrel{\gamma^{\prime}}{\rightarrow} K(\mathcal{D}(M)) /(K(\partial M) \oplus K(M)) .
$$

To an isomorphism $\sigma$ (see $(\overline{36}))$ that is independent of $\xi$ over a neighborhood of the boundary $\partial M$, this map assigns a classical boundary value problem (2) in the following way. For the symbol $\sigma$ we construct an elliptic first-order pseudodifferential operator that has the form

$$
D=\sigma \circ \Lambda
$$

near the boundary, where $\sigma=\sigma(x)$ is a homomorphism of vector bundles and $\Lambda$ has the principal symbol $|\xi|$. It remains to modify the operator $D$ near the boundary, as it was done in Example 1:

$$
D^{\prime}=\sigma \circ\left((1-\chi(t)) \Lambda+\chi(t)\left(-\frac{\partial}{\partial t}+\Lambda_{+}\right)\right) .
$$

The operator $\Lambda_{+}$here has the principal symbol $\left|\xi^{\prime}\right|$. Finally, we define

$$
\gamma^{\prime}(\sigma) \stackrel{\text { def }}{=}\left[D^{\prime}\right] \in K(\mathcal{D}(M)) /(K(\partial M) \oplus K(M)) \text {. }
$$

This is well defined, since the operator $D^{\prime}$ defines a Fredholm boundary value problem without boundary conditions.

Remark 5 In terms of the isomorphism (38) it is easy to prove the following index formula for classical boundary value problems of the form (2):

$$
\text { ind }(D, 1)=p_{!} \gamma(D, 1), \quad p: \stackrel{\circ}{M} \rightarrow p t,
$$

where $p_{!}: K_{c}\left(T^{*} \stackrel{\circ}{M}\right) \rightarrow K(p t)=\mathbf{Z}$ is the direct image in $K$-theory. 
Proof. Indeed, consider a boundary value problem $\left(D^{\prime}, 1\right)$ on the manifold $M$ that coincides with the original problem $(D, 1)$ in a neighborhood of the boundary and on the whole manifold is a boundary value problem from Example 1. Let us examine the composition

$$
D_{0}=\left(D^{\prime}, 1\right)^{-1} \circ(D, 1) .
$$

Its principal symbol $\sigma\left(D_{0}\right)$ is the identity isomorphism over a neighborhood of the boundary $\partial M$. Thus, the index of the operator $D_{0}$ can be computed by the Atiyah-Singer formula

$$
\operatorname{ind} D_{0}=p_{!}\left[\sigma\left(D_{0}\right)\right], \quad\left[\sigma\left(D_{0}\right)\right] \in K_{c}\left(T^{*} \stackrel{\circ}{M}\right) .
$$

The boundary value problem $\left(D^{\prime}, 1\right)$, however, has index zero and also defines the trivial element in the $K$-group:

$$
\gamma\left(D^{\prime}, 1\right)=0 \in K_{c}\left(T^{*} \stackrel{\circ}{M}\right) .
$$

Hence, we obtain the desired formula

$$
\text { ind }(D, 1)=\operatorname{ind} D_{0}=p_{!}\left[\sigma\left(D_{0}\right)\right]=p_{!} \gamma(D, 1) \text {. }
$$

Remark 6 The reductions of the previous section give an isomorphism of the group (35) and the group of stable homotopy classes of classical boundary value problems (1). In this way, considering spectral boundary value problems (2), we make no loss of generality and cover the general case as well.

The three groups introduced above are related by an exact sequence.

Proposition 2 The sequence

$$
K_{c}\left(T^{*} \stackrel{\circ}{M}\right) \stackrel{\alpha}{\rightarrow} K\left(\mathcal{D}_{e v}(M)\right) /(K(\partial M) \oplus K(M)) \stackrel{\beta}{\rightarrow} K\left(P_{e v}(\partial M)\right) / K(\partial M)
$$

is exact. Here $\alpha$ is induced by the embedding of classical boundary value problems into even ones and the map $\beta$ is induced by the forgetful map

$$
(D, P) \longrightarrow P \text {. }
$$

Proof. The equality $\beta \circ \alpha=0$ is obvious, since the projection in the classical boundary value problem is the unit projection and hence defines the trivial element in the group $K\left(P_{e v}(\partial M)\right) / K(\partial M)$.

Let us now verify the inclusion $\operatorname{ker} \beta \subset \operatorname{Im} \alpha$. Suppose that for an even boundary value problem $(D, P)$ one has

$$
\beta[D, P]=0 \in K\left(P_{e v}(X)\right) / K(X) .
$$


This means that there exists a homotopy of even projections that connects the projection $P$ and the projection on the space of sections of a vector bundle on $\partial M$, denoted by $P^{\prime}=P_{C^{\infty}\left(\partial M, G^{\prime}\right)}$. Let us denote this homotopy by $P_{t}: P_{0}=0, P_{1}=P^{\prime}$, and lift this homotopy of projections to a homotopy of spectral boundary value problems $\left(D_{t}, P_{t}\right)$. In the Grothendieck group $K\left(\mathcal{D}_{e v}(M)\right) /(K(\partial M) \oplus K(M))$, we have

$$
[D, P]=\left[D_{1}, P_{1}\right]=\alpha\left[D_{1}, 1_{C^{\infty}\left(\partial M, G^{\prime}\right)}\right],
$$

since the boundary value problem $\left(D_{1}, P_{1}\right)$ is classical.

This establishes the exactness of the sequence (39).

From now on we assume that the manifold $M$ is even-dimensional. The third term in the sequence (39) is simplified in this case: according to (12), finite-dimensional projections generate a subgroup in this term that is isomorphic to $\mathbf{Z}$

$$
\mathbf{Z} \subset K\left(P_{e v}(X)\right) / K(X),
$$

and the quotient group

$$
K\left(P_{e v}(X)\right) /(K(X) \oplus \mathbf{Z}) \simeq K\left(P^{*} X\right) / K(X), \quad P^{*} X \text { is the projectivization of } S^{*} X,
$$

according to [8], consists of elements of finite orders that are powers of 2 .

In a similar fashion, in the Grothendieck group of even boundary value problems there is a subgroup

$$
\mathbf{Z} \subset K\left(\mathcal{D}_{e v}(M)\right) /(K(\partial M) \oplus K(M)),
$$

which is generated by boundary value problems for zero operators with finite rank projections on the right-hand sides. This enables us to refine the sequence (39):

$$
K_{c}\left(T^{*} \stackrel{\circ}{M}\right) \stackrel{\alpha}{\rightarrow} K\left(\mathcal{D}_{e v}(M)\right) /(K(\partial M) \oplus K(M) \oplus \mathbf{Z}) \stackrel{\beta}{\rightarrow} K\left(P^{*}(\partial M)\right) / K(\partial M) .
$$

For an even boundary value problem, we define an analog of the topological index of Atiyah-Singer. Let us consider the double

$$
2 M \stackrel{\text { def }}{=} M \bigcup_{\partial M} M
$$

of the manifold $M$. On this manifold we have the (continuous) elliptic symbol

$$
\sigma(\tilde{D})(\xi)=\sigma(D)(\xi) \cup \sigma(D)(-\xi),
$$

on the first copy of the manifold this symbol is equal to the original symbol $\sigma(D)(\xi)$, and on the second copy it is $\sigma(D)(-\xi)$. The continuity at the place of gluing follows from the equality

$$
\sigma(D)\left(-\tau, \xi^{\prime}\right)=\sigma(D)\left(-\tau,-\xi^{\prime}\right)
$$

In this notation the following index formula for spectral boundary value problems is valid. 


\section{Theorem 5}

$$
\text { ind }(D, P)=\frac{1}{2} \operatorname{ind}_{t}(\sigma(\tilde{D}))-d(P),
$$

where $d(P)$ is the dimension of the projection $P$ for the trivial normalization (see Theorem 1).

Proof. The index of the boundary value problem and the right-hand side of (41) extend to homomorphisms of abelian groups, denoted, respectively, by

$$
\text { ind, ind }{ }^{\prime}: K\left(\mathcal{D}_{e v}(M)\right) /(K(\partial M) \oplus K(M)) \longrightarrow \mathbf{Q} .
$$

Let us take advantage of the exact sequence (40). We first check that the two homomorphisms coincide for the classical boundary value problems. In this case we obtain $d(P)=0$, since we have the unit projection and the normalization $\chi$ is taken to be trivial. Hence, it remains to verify the validity of the index formula for the classical boundary value problems

$$
\text { ind }(D, 1)=\frac{1}{2} \operatorname{ind}_{t}(\sigma(D)(\xi) \cup \sigma(D)(-\xi)) \text {. }
$$

By virtue of the isomorphism (38), the left- and right-hand sides of (42) are homomorphisms of groups

$$
K_{c}\left(T^{*} \stackrel{\circ}{M}\right) \longrightarrow \mathbf{Q} .
$$

The right-hand side of (42) is decomposed for the classical boundary value problems into two terms

$$
\begin{gathered}
\operatorname{ind}_{t}(\sigma(D)(\xi) \cup \sigma(D)(-\xi))=\operatorname{ind}_{t}(\sigma(D)(\xi))+\operatorname{ind}_{t}(\sigma(D)(-\xi)), \\
\sigma(D)(\xi), \sigma(D)(-\xi) \in K_{c}\left(T^{*} \stackrel{\circ}{M}\right) .
\end{gathered}
$$

Let us show that on an even-dimensional manifold the two terms in the last formula are equal. Indeed, in the cohomological form we obtain

$$
\operatorname{ind}_{t}(\sigma(D)(-\xi))=\left\langle\operatorname{ch}[\sigma(D)(-\xi)] \operatorname{Td}\left(T^{*} M \otimes\right),\left[T^{*} \stackrel{\circ}{M}\right]\right\rangle .
$$

The involution on the space $T^{*} M$

$$
\xi \longrightarrow-\xi
$$

preserves its orientation. Then from (43) we obtain

$$
\operatorname{ind}_{t}(\sigma(D)(\xi))=\operatorname{ind}_{t}(\sigma(D)(-\xi)) .
$$

The equality in (42) now follows from the index formula for the classical boundary value problems (see Remark 5):

$$
\text { ind }(D, 1)=\operatorname{ind}_{t}(\sigma(D)(\xi)),
$$


The homomorphisms ind and ind' obviously coincide on the subgroup $\mathbf{Z}$ generated by boundary value problems for operators equal to zero. In this way, the difference ind - ind' descends to a homomorphism of the quotient group

$$
\text { ind }- \text { ind }^{\prime}:\left[K\left(\mathcal{D}_{e v}(M)\right) /(K(\partial M) \oplus K(M) \oplus \mathbf{Z})\right] / \operatorname{Im} \alpha \rightarrow \mathbf{Q} .
$$

The exactness of the sequence (40) implies $\operatorname{Im} \alpha=\operatorname{ker} \beta$. Let us now note that the homomorphism $\beta$ takes values in the torsion group (see the sequence (40)). Thus, the difference ind - ind' cannot be nontrivial, since it is defined on the group consisting of elements of a finite order.

The index formula is thereby proved.

Operators in subspaces on closed manifolds and boundary value problems on manifolds with boundary studied above are related.

Let $D$ be an operator in subspaces, on a closed manifold $M$

$$
D: \operatorname{Im} P_{1} \rightarrow \operatorname{Im} P_{2}, \quad \operatorname{Im} P_{1,2} \subset C^{\infty}\left(M, E_{1,2}\right) .
$$

Let us assign an elliptic boundary value problem to this operator.

On the cylinder $M \times[0,1]$, consider an elliptic first-order operator with constant coefficients along the cylinder

$$
D^{\prime}=\frac{\partial}{\partial t}+\left(1-P_{1}\right) \Lambda\left(1-P_{1}\right)-P_{1} \Lambda P_{1}: C^{\infty}\left(M \times[0,1], E_{1}\right) \rightarrow C^{\infty}\left(M \times[0,1], E_{1}\right),
$$

where $\Lambda$ is a positive self-adjoint operator of the first order on $M$ with principal symbol $|\xi|$. On the two bases of the cylinder we impose the following boundary conditions: one boundary condition spectral, while the other is general,

$$
\left\{\begin{array}{c}
\left(1-P_{1}\right) u(0)=g \in \operatorname{Im}\left(1-P_{1}\right) \subset C^{\infty}\left(M, E_{1}\right) \\
D u(1)=g^{\prime} \in \operatorname{Im} P_{2} \subset C^{\infty}\left(M, E_{2}\right)
\end{array}\right.
$$

Let us note that the ellipticity of the boundary conditions (46) is equivalent to the ellipticity of the operator in the subspaces (44).

Proposition 3 The index of the boundary value problem (45), (46) is equal to the index of the operator in subspaces (44).

Proof. First, it suffices to prove this proposition for the case in which the projections $P_{1,2}$ are orthogonal. In this situation the operator (45) has the form

$$
D^{\prime}=\frac{\partial}{\partial t}+A, \quad A \text { is a self-adjoint operator, }
$$

which makes it possible to reduce the proof of the equality of the indices to a direct calculation (with the help of the eigenfunctions of $A$ ). These calculations are omitted. 


\section{$6 \quad$ Eta invariants and even projections}

Following [8], we say that a classical pseudodifferential operator $A$ of integer positive order $m$ is admissible if its complete symbol

$$
a(x, \xi) \sim a_{m}(x, \xi)+a_{m-1}(x, \xi)+\ldots
$$

satisfies the parity conditions

$$
a_{\alpha}(x,-\xi)=(-1)^{\alpha} a_{\alpha}(x, \xi), \quad \forall \xi \neq 0, \quad x, \quad \alpha=m, m-1, m-2, \ldots
$$

(the admissibility of an operator is independent of the choice of a coordinate system where the complete symbol is considered). We also recall that the $\eta$-function of a self-adjoint operator $A$ is defined as

$$
\eta(s, A)=\sum_{\lambda \in \operatorname{spec} A} \operatorname{sign} \lambda|\lambda|^{-s}
$$

where the sum is taken over the nonzero eigenvalues of the operator $A$ (with regard to their multiplicities). It is well known that for an elliptic self-adjoint operator on an odddimensional manifold the $\eta$-invariant of Atiyah-Patodi-Singer [3], which is by definition equal to the value at the origin of the analytic continuation of the $\eta$-function, is a finite number, i.e., the $\eta$-function does not have a pole at the origin. Assuming additionally that $A$ is an admissible operator of even order on an odd-dimensional manifold, one can claim that the reduction of the $\eta$-invariant modulo $\mathbf{Z}$ is invariant under deformations of the operator, while the integer jumps occur as a result of (discontinuous) changes of the nonnegative spectral subspace of the operator.

Such a homotopy invariance suggests that for this class of operators the $\eta$-invariant of the operator $A$ is completely determined by its nonnegative spectral subspace (this subspace is, actually, the range of an even projection). This idea is realized in the following proposition.

Proposition 4 Let $P_{+}$be an even pseudodifferential projection that is equal to a nonnegative spectral projection for some admissible operator $A$. Then the dimension of the projection with the trivial normalization $\chi: K(M) \rightarrow \mathbf{R}$ and the $\eta$-invariant of the operator $A$ are equal:

$$
d\left(P_{+}\right)=\frac{\eta(0, A)+\operatorname{dim} \operatorname{ker} A}{2} \stackrel{\text { def }}{=} \eta(A)
$$

Proof. The orders of the elements of the group $K\left(P^{*} M\right) / K(M)$ are powers of 2 . Hence, (see Sec. 1 and the paper [8]) for sufficiently large $N$ the operator $2^{N} A$ is homotopic (in the space of admissible self-adjoint elliptic operators) to some operator, denoted by $A^{\prime}$, which is equal to a direct sum of a positive and a negative admissible operator. Hence, we obtain $\eta\left(A^{\prime}\right)=0$ (see [8]). Let us denote an arbitrary homotopy of this form by $A_{t}$. 
Recalling that the spectral flow of the family $A_{t}$ through the point zero is equal to the net number of jumps of the $\eta$-invariant, we obtain

$$
\eta\left(2^{N} A\right)=-\operatorname{sf} A_{t}
$$

On the other hand, by considering a spectral section (see [10]) $P_{t}$ for the family $A_{t}$, we can show (this is one of the definitions of the spectral flow) that

$$
\operatorname{sf} A_{t}=-\operatorname{ind}\left(2^{N} P_{+}, P_{0}\right)+\operatorname{ind}\left(P_{+, A^{\prime}}, P_{1}\right) .
$$

The last expression is equal to the $d$-dimension (see Remark 1)

$$
-\operatorname{ind}\left(2^{N} P_{+}, P_{0}\right)+\operatorname{ind}\left(P_{+, A^{\prime}}, P_{1}\right)=-d\left(2^{N} P_{+}\right)+d\left(P_{+, A^{\prime}}\right)=-d\left(2^{N} P_{+}\right) .
$$

We finally obtain the desired formula

$$
\eta(A)=\frac{1}{2^{N}} \eta\left(2^{N} A\right)=\frac{1}{2^{N}} d\left(2^{N} P_{+}\right)=d\left(P_{+}\right) .
$$

Remark 7 An even projection $P$ satisfies the conditions of Proposition 4 if and only if it is an admissible operator of order zero itself.

Indeed, the admissibility of the operator $A$ implies the admissibility of its spectral projection (see [8]). To prove the converse statement, let us note that admissible operators form an algebra. Consider an elliptic operator defined by the formula

$$
A=P \Delta P-(1-P) \Delta(1-P),
$$

where $\Delta$ denotes an arbitrary admissible positive self-adjoint operator of order two with the principal symbol of the Laplacian $|\xi|^{2}$. The operator $A$ is exactly the desired one (i.e. an operator with nonnegative spectral projection equal to $P$ ). This completes the proof of the remark.

In the paper [8], the topological meaning of the invariant $\eta \bmod \mathbf{Z}$ (i.e. $\operatorname{modulo}$ jumps) was considered. It was noticed that this reduction defines a homomorphism

$$
\eta: K\left(P^{*} M\right) / K(M) \longrightarrow \mathbf{Z}\left[\frac{1}{2}\right] \bmod \mathbf{Z}
$$

By virtue of Proposition \&, the obtained index formula for operators in subspaces (15), when reduced $\bmod \mathbf{Z}$, gives a new formula for the $\eta$-invariant, [] which we state now as a corollary.

\footnotetext{
${ }^{9}$ But not for all elements with principal symbols in the group $K\left(P^{*} M\right)$.
} 


\section{Corollary 1 Let}

$$
W \in \operatorname{ker}\left\{\pi^{*}: K\left(P^{*} M\right) / K(M) \longrightarrow K\left(S^{*} M\right) / K(M)\right\}, \quad \pi: S^{*} M \rightarrow P^{*} M .
$$

Then the fractional part of the $\eta$-invariant of the bundle $W$ is the half-integer

$$
\eta(W) \equiv \frac{1}{2} \operatorname{ind}_{t}\left(\sigma(\xi) \sigma^{-1}(-\xi): \pi_{S}^{*} F \rightarrow \pi_{S}^{*} F\right) \bmod \mathbf{Z}, \quad \pi_{S}: S^{*} M \rightarrow M, F \in \operatorname{Vect}(M),
$$

where $\sigma$ is an arbitrary isomorphism of vector bundles $\pi^{*} W$ and $\pi_{S}^{*} F$ over the cotangent sphere bundle, which exists by condition (47).

The index formula for even boundary value problems leads to a similar formula (cf. [18]).

Corollary 2 Let $(D, B, P)$ be an even elliptic boundary value problem on the manifold $M$. Then the fractional part of the $\eta$-invariant of the even bundle $\operatorname{Im} \sigma(P)$ is a halfinteger and can be computed by the formula

$$
\eta(\operatorname{Im} \sigma(P)) \equiv \frac{1}{2} \operatorname{ind}_{t}(\sigma(D)(\xi) \cup \sigma(D)(-\xi)) \bmod \mathbf{Z}
$$

The last statement gives the cobordism invariance, which we state now in terms of $K$-theory. Let us recall some constructions from [12].

Consider the homomorphism

$$
\begin{array}{ccc}
K\left(S^{*} X\right) / K(X) & \longrightarrow & K_{c}\left(T^{*} X \oplus 1\right) \\
W=\operatorname{Im} P \subset \pi^{*} E & \longrightarrow & {[\tau+i(2 P-1)|\xi|]}
\end{array}
$$

where $E \in \operatorname{Vect}(X), \pi: S^{*} X \rightarrow X$ is the natural projection and the element

$$
[\tau+i(2 P-1)|\xi|] \in K_{c}\left(T^{*} X \oplus 1\right)
$$

is understood in the sense of difference construction, i.e. outside of the zero section of the bundle $T^{*} X \oplus 1$ it defines an isomorphism in the pullback of the bundle $E$ on the space $T^{*} X \oplus 1$. It can be shown (see [12]) that this homomorphism coincides with the composition

$$
K\left(S^{*} X\right) / K(X) \stackrel{\delta}{\rightarrow} K^{1}\left(T^{*} X\right)=K_{c}\left(T^{*} X \times \mathbf{R}\right),
$$

where $\delta$ is induced by the coboundary operator in $K$-theory. Moreover, the map $\delta$ is an isomorphism (for an odd-dimensional manifold $X$ this is easy to show by using a nonvanishing vector field). Making use of the identification (50) given by formula (49), we obtain the desired cobordism invariance in the following form. 
Corollary 3 On the boundary $X=\partial M$ of a smooth even-dimensional manifold $M$ consider an even subbundle $[W] \in K\left(P^{*} X\right) / K(X)$ and its pullback to the bundle of cotangent spheres,

$$
\pi^{*}[W] \in K\left(S^{*} X\right) / K(X), \quad \pi: S^{*} X \rightarrow P^{*} X .
$$

Suppose that this extends inside the manifold $M$, i.e. it lies in the range of the restriction operator

$$
\begin{aligned}
i^{*}: K_{c}\left(T^{*} M\right) \rightarrow & K_{c}\left(\left.T^{*} M\right|_{X}\right),\left.\quad T^{*} M\right|_{X} \subset T^{*} M, \\
& \pi^{*}[W] \in \operatorname{Im} i^{*}
\end{aligned}
$$

for the identification (50). Then the doubled $\eta$-invariant of the bundle $W$ is an integer:

$$
2 \eta(W) \bmod \mathbf{Z}=0 .
$$

\section{Examples}

1. On a closed, connected, odd-dimensional, oriented, Riemannian manifold $M$ consider an elliptic self-adjoint differential operator of second order acting on exterior 1-forms by the formula

$$
A=d \delta-\delta d: \Lambda^{1}(M) \longrightarrow \Lambda^{1}(M),
$$

where $d$ is the usual exterior derivative and $\delta$ is the adjoint operator. A direct calculation shows that the principal symbol of the nonnegative spectral projection $P$ for this operator at a point $\xi \neq 0$ is a projection on the line generated by the covector $\xi$ itself. In other words, the projection $P$ is an even projection, while the image of the principal symbol $\operatorname{Im} \sigma^{\prime}(P)$ on each of the projective spaces $P_{x}^{*} M$ is exactly the tautological line bundle, which is known to be nontrivial. At the same time, the pullback of this bundle to the cosphere bundle is already trivial with the natural trivialization

$$
\begin{aligned}
\kappa: \operatorname{Im} \sigma(P) & \longrightarrow \mathbf{C}, \\
\kappa(x, \xi, \eta) & =(\xi, \eta),
\end{aligned}
$$

where $(\xi, \eta)$ is the inner product of two proportional vectors. The calculation made in the paper [8] shows that in this case

$$
\eta(A)=\left.\operatorname{dim} \operatorname{ker}(d \delta+\delta d)\right|_{\Lambda^{1}(M)}-1=\operatorname{dim} H^{1}(M)-1 .
$$

Hence, for an elliptic operator

$$
D: \operatorname{Im} P \longrightarrow C^{\infty}(M)
$$

acting in the corresponding subspaces, the index formula can be rewritten in the form

$$
\text { ind }\left(D, P, P_{C^{\infty}(M)}\right)=\operatorname{ind}_{t}\left(\sigma(D) \kappa^{-1}: \pi^{*} \mathbf{C} \rightarrow \pi^{*} \mathbf{C}\right)+\operatorname{dim} H^{1}(M)-1,
$$


where

$$
\operatorname{ind}_{t}\left(\sigma(D) \kappa^{-1}: \pi^{*} \mathbf{C} \rightarrow \pi^{*} \mathbf{C}\right)
$$

is the topological index of a usual elliptic pseudodifferential operator acting on functions. IV 2. An operator similar to the one from the previous example is known for the case of coefficients in a bundle $W \in \operatorname{Vect}(M)$ and also for the case of forms of higher degrees. Namely, consider a vector bundle $W$ with a connection $D$. The corresponding operator is defined by the formula

$$
A \otimes 1_{W}=D D^{*}-D^{*} D: \Lambda^{k}(M, W) \longrightarrow \Lambda^{k}(M, W) .
$$

It is an elliptic self-adjoint operator. Its principal symbol is the tensor product

$$
\sigma\left(A \otimes 1_{W}\right)=\sigma(A) \otimes 1_{W}
$$

In Gilkey's paper [8], the problem of computation of the fractional part of the $\eta$-invariant for operators of the above type was posed. It follows from the formula (48), for example, that on a parallelizable manifold the fractional part of such operators acting on 1-forms with coefficients in an arbitrary bundle $W$ is zero. Indeed, by the index formula it is equal to half the index of an operator with a constant principal symbol.

The problem posed by Gilkey asks for an operator with a nonzero fractional part of the $\eta$-invariant. It remains open. In the context of even projections this problem can be partly restated as follows: is it possible that fractional terms appear in our index formulas?

\section{References}

[1] M.F. Atiyah and R. Bott. The index problem for manifolds with boundary. In Bombay Colloquium on Differential Analysis, 1964, pages 175-186, Oxford. Oxford University Press.

[2] B.-W. Schulze, B. Sternin, and V. Shatalov. On general boundary value problems for elliptic equations. Math. Sb., 189, No. 10, 1998, 1573-1586.

[3] M. Atiyah, V. Patodi, and I. Singer. Spectral asymmetry and Riemannian geometry I. Math. Proc. Cambridge Philos. Soc., 77, 1975, 43-69.

[4] A. Savin, B.-W. Schulze, and B. Sternin. On the invariant index formulas for spectral boundary value problems. Differentsial'nye uravnenija, 35, No. 5, 1999, 705-714. [Russian].

\footnotetext{
${ }^{10}$ For $\operatorname{dim} M \geq 3$ this index is well known to be zero. In order to obtain a nontrivial index in this situation, one can consider matrix operators.
} 
[5] A.Yu. Savin. On the index formula splitting for spectral boundary value problems. Dokladi RAN. (in print).

[6] L. Hörmander. The Analysis of Linear Partial Differential Operators. III. SpringerVerlag, Berlin Heidelberg New York Tokyo, 1985.

[7] V. Nazaikinskii, B.-W. Schulze, B. Sternin, and V. Shatalov. Spectral boundary value problems and elliptic equations on singular manifolds. Differents. Uravnenija, 34, No. 5, 1998, 695-708. [Russian].

[8] P.B. Gilkey. The eta invariant of even order operators. Lecture Notes in Mathematics, 1410, 1989, 202-211.

[9] M.S. Birman and M.Z. Solomjak. On the subspaces admitting a pseudodifferential projection. Vestnik $L G U$, No. 1, 1982, $18-25$.

[10] R. Melrose and P. Piazza. Families of Dirac operators, boundaries and the $b$-calculus. J. of Diff. Geom., 46, No. 1, 1997, 99-180.

[11] K. Wojciechowski. A note on the space of pseudodifferential projections with the same principal symbol. J. Operator Theory, 15, No. 2, 1986, 207-216.

[12] M. Atiyah, V. Patodi, and I. Singer. Spectral asymmetry and Riemannian geometry III. Math. Proc. Cambridge Philos. Soc., 79, 1976, 71-99.

[13] X. Dai and W. Zhang. Splitting of the family index. Comm. Math. Phys., 182, No. 2, 1996, 303-318.

[14] M.F. Atiyah, R. Bott, and V.K. Patodi. On the heat equation and the index theorem. Invent. Math., 19, 1973, 279 - 330.

[15] M.F. Atiyah and I.M. Singer. The index of elliptic operators I. Ann. Math., 87, 1968, 484-530.

[16] S. Rempel and B.-W.Schulze. Index Theory of Elliptic Boundary Problems. Akademie-Verlag, Berlin, 1982.

[17] M. Agranovich and M. Vishik. Elliptic problems with parameter and parabolic problems of general type. Uspekhi Mat. Nauk, 19, No. 3, 1964, 53-161. English transl.: Russ. Math. Surv. 19 (1964), N 3, p. 53-157.

[18] P.B. Gilkey. The eta invariant for even dimensional Pin ${ }^{c}$ manifolds. Advances in Mathematics, 58, 1985, 243-284.

Moscow State University 